\title{
Toward Unconstrained Fingerprint Recognition: a Fully Touchless 3-D System Based on Two Views on the Move
}

\author{
Ruggero Donida Labati, Member, IEEE, Angelo Genovese, Member, IEEE, \\ Vincenzo Piuri, Fellow, IEEE, and Fabio Scotti, Senior Member, IEEE
}

\begin{abstract}
Touchless fingerprint recognition systems do not require contact of the finger with any acquisition surface and thus provide an increased level of hygiene, usability and user acceptability of fingerprint-based biometric technologies. The most accurate touchless approaches compute three-dimensional models of the fingertip. However, a relevant drawback of these systems is that they usually require constrained and highly cooperative acquisition methods. We present a novel, fully touchless fingerprint recognition system based on the computation of threedimensional models. It adopts an innovative and less-constrained acquisition setup compared with other previously reported threedimensional systems, does not require contact with any surface or a finger placement guide, and simultaneously captures multiple images while the finger is moving. To compensate for possible differences in finger placement, we propose novel algorithms for computing three-dimensional models of the shape of a finger. Moreover, we present a new matching strategy based on the computation of multiple touch-compatible images. We evaluated different aspects of the biometric system: acceptability, usability, recognition performance, robustness to environmental conditions and finger misplacements, compatibility and interoperability with touch-based technologies. The proposed system proved to be more acceptable and usable than touch-based techniques. Moreover, the system displayed satisfactory accuracy, achieving an Equal Error Rate (EER) of $0.06 \%$ on a dataset of 2,368 samples acquired in a single session and $0.22 \%$ on a dataset of 2,368 samples acquired over the course of one year. The system was also robust to environmental conditions and to a wide range of finger rotations. The compatibility and interoperability with touch-based technologies was greater or comparable to those reported in public tests using commercial touchless devices.
\end{abstract}

Index Terms-Fingerprint, 3D reconstruction, touchless, contactless, biometrics, less-constrained, on the move.

\section{INTRODUCTION}

$\mathbf{F}$ INGERPRINT recognition systems are biometric technologies that are widely used in a variety of application scenarios $[1,2]$. These typically feature touch-based acquisition devices, such as optical and solid-state sensors. However, these systems suffer from various problems [3-5]:

- distortions in the acquired samples due to elastic deformations resulting from friction with the skin of the finger;

- low-contrast regions in the fingerprint images due to skin conditions, humidity, and dirt on the sensor plate;

- release of latent fingerprints.

Ruggero Donida Labati, Angelo Genovese, Vincenzo Piuri, and Fabio Scotti are with the Department of Computer Science, Università degli Studi di Milano, via Bramante, 65, I-26013 Crema (CR), Italy, e-mail: (ruggero.donida, angelo.genovese, vincenzo.piuri, fabio.scotti)@unimi.it.
Touchless fingerprint recognition systems are emerging technologies designed to overcome these problems by avoiding contact of the fingertip with any acquisition surface. Moreover, touchless systems can increase the user acceptability of fingerprint biometrics, which is currently limited by cultural factors and fears related to the transmission of skin diseases. Touchless technologies can also reduce acquisition time and user training requirements.

However, the recognition accuracy and interoperability of touchless systems are usually lower than those of touch-based systems and are technologies based on more constrained, expensive and bulkier acquisition devices. Recent work in this field aims to reduce such limitations.

Touchless systems based on three-dimensional models usually obtain better accuracy compared with techniques based on single images because they are more robust to perspective distortions and can extract additional features from threedimensional shapes.

Recent work has examined biometric techniques based on touchless acquisitions and three-dimensional models for handbased traits, such as: hand geometry [6,7], palmprint [8-11], finger geometry [12], and knuckle-print $[13,14]$. Other studies have investigated the reduction of acquisition constraints in systems based on traits traditionally acquired in a touchless manner, such as face [15-19], iris [20-22], gait [23], and ear [24].

In touchless systems based on finger and hand characteristics, samples acquired without controlling the user's posture may exhibit low quality, perspective distortions, and nonuniform resolution. Therefore, most biometric systems use guides to place the body part. A few studies on techniques that do not use placement guides have been performed on traits such as fingerprints [25-27], hand surface [7] and finger shape [28]. Studies of fingerprint recognition with movement have been limited to two-dimensional acquisitions [29].

The present study presents a novel fully touchless fingerprint recognition system based on the computation of threedimensional models from two-view images captured during movement. The system is less-constrained [30] than the threedimensional recognition technologies reported in the literature, and it requires only a minimum level of user cooperation because the acquisitions are performed while the finger is moving, in a single instant of time and without contact with any acquisition surface or finger placement guide. Moreover, the finger can be placed with different three-dimensional 
orientations as long as a sufficient area of the ridge pattern is visible by the cameras. The proposed system also presents important advantages in usability and user acceptance. Fig. 1 shows an example of the biometric acquisition process.

We used a multiple-view technique because it permits to capture of the information needed to compute a threedimensional model in a single instant of time, thus reducing constraints in finger positioning. Other methods, such as structured light and photometric stereo techniques, capture multiple frames and require placement guides to avoid possible artifacts due to micro movements of the finger.

Our system cannot compute the three-dimensional depth of ridges and valleys but reconstructs a metric representation (in $\mathrm{mm}$ ) of the finger's shape that is invariant to the angle and distance of the finger to the cameras. Next, it computes a touch-compatible image from the three-dimensional model and reduces non-idealities due to less-constrained acquisitions using novel strategies for feature extraction and matching.

In contrast to three-dimensional technologies reported in the literature, which have the main goal of increasing the accuracy of fingerprint recognition technologies, our system aims to increase the usability and user acceptability of fingerprint biometric techniques by performing less-constrained acquisitions and providing recognition accuracy comparable to touch-based technologies. Our goal is to move toward the creation of technologies for fingerprint recognition that are able to perform identity verifications on-the move with high usability. These technologies should also be integrable in ambient intelligence applications $[31,32]$ that require biometrics to verify the identity of the users [33] or require accurate identity verification before performing continuous identity recognition [34].

Because the proposed biometric system avoids any contact with the sensor, it also presents advantages in hygiene and privacy [35] compared with touch-based fingerprint biometrics because no latent fingerprints are left during the acquisition process. Our system can therefore be considered an alternative to touch-based technologies for application contexts in which usability, privacy and hygiene are important concerns (e.g., hospitals, stadiums and public buildings).

The contributions of the present study are twofold. First, we propose an innovative acquisition setup and new processing algorithms that introduce important novelties compared with previous studies $[25,36]$. Second, we present direct comparisons between the proposed fully touchless systems, touchless approaches in the literature, and touch-based technologies. We performed extensive tests on new extended scenario-designed databases [37] in which results from the same users were acquired using both touch-based and touchless technologies. The comparative evaluations encompass different characteristics of the systems: recognition accuracy under different operation conditions; computational time; compatibility and interoperability between biometric systems, and user acceptance.

The paper is structured as follows. Section II presents a literature review of touchless fingerprint recognition systems. Section III details the proposed system. Section IV describes the experiments performed and the obtained results. Finally, Section V summarizes the work.

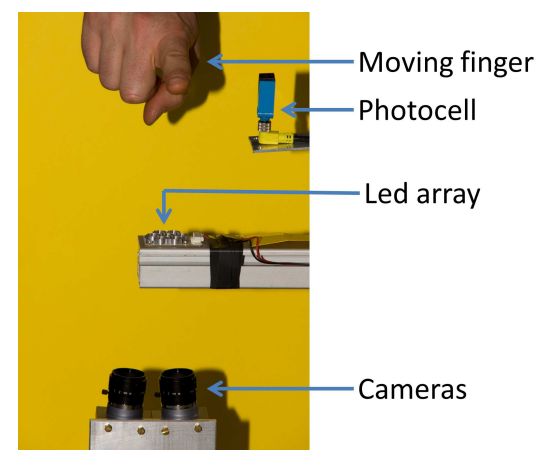

Fig. 1. Example of the biometric acquisition process. The acquisition is based on two-view images captured simultaneously during finger movement and without support guides.

\section{Previous Work}

\section{A. Two-dimensional systems}

The simplest acquisition techniques adopted by touchless fingerprint recognition systems use a low-cost digital camera in uncontrolled light conditions [38-40]. The obtained images, however, display poor contrast between ridges and valleys. For this reason, specific illumination techniques (e.g., a point light source) are frequently adopted to improve the visibility of the fingertip details [41-44]. However, white and infrared light wavelengths tend to penetrate the skin and be absorbed by the epidermis, producing relatively low-contrast images $[4,45]$. A blue light with a wavelength of $500 \mathrm{~nm}$ permits better-quality images [46,47]. Other acquisition systems use illumination methods that exploit the optical characteristics inside of the finger [48].

Fingerprint images obtained by performing single-view acquisitions typically display non-uniform resolution and unfocused regions. To reduce these problems, some systems compute a mosaic of multiple views $[49,50]$ or use ring mirrors [47].

Images captured by touchless sensors cannot be directly used by recognition methods designed for touch-based fingerprint samples [40,51]. Consequently, most previously reported systems compute touch-compatible images by applying enhancement algorithms and resolution normalization techniques. These methods usually perform background subtraction, noise reduction $[38,41-43,52]$, and enhancement of the ridge pattern [53]. In some cases, the focal length and the distance between the finger and camera are used to normalize the image resolution $[4,44]$, but estimating these parameters becomes critical for the final accuracy. Unconstrained acquisition systems only perform an approximated normalization by evaluating the finger's silhouette [38]. Preprocessing methods for touchless images captured by mobile devices are reviewed in [54].

Most systems perform matching of touchless fingerprint samples using methods based on the distance between minutia points $[4,38,49]$, which requires samples with constant and known resolution. Other systems use matching methods based on adimensional features $[55,56]$ and use matchers based on computational intelligence techniques [57]. Studies of quality assessment methods [26,27], core detection algorithms [58], 
techniques designed for multiple fingers [59], compression of touchless fingerprint images [60], acquisition systems with vitality checks $[47,61]$, and recognition techniques for lowresolution images of fingers $[62,63]$ have been reported.

Both commercial two-dimensional touchless devices that use finger placement guides [64-67] and commercial technologies that do not use any guide [68,69] exist. However, their accuracy has not yet been documented.

\section{B. Three-dimensional systems}

All previously reported methods for computing threedimensional fingerprint models use finger placement guides. These methods are based on multiple views [70-73], structured light approaches [74-77], photometric stereo systems [78,79], depth from focus methods [80], or acoustic imaging [81]..

The system described in [70-72] uses an acquisition setup composed of five cameras and a set of green LEDs located in a semicircle around the finger. It estimates the finger volume using a shape from silhouette technique. It then applies a triangulation algorithm to corresponding pairs of points to compute the three-dimensional shape of the finger. A depth map and a grayscale image of the ridge pattern compose the three-dimensional model describing the fingerprint. Similarly, the system presented in [73] uses a multiple-view setup comprising three cameras and three LEDs to reconstruct a three-dimensional model of the finger shape volume.

A system based on a structured light technique is presented in $[74,75]$. The system is capable of estimating both the three-dimensional depths of ridges and valleys and the texture of the ridge pattern. The three-dimensional reconstruction is performed by projecting a sine-wave pattern shifted several times and by evaluating the phase shift of the projected pattern in every captured frame. The texture of the ridge pattern is computed as the albedo image [82]. The structured light techniques presented in [76,77] compute three-dimensional models of the finger shape with a superimposed texture of the ridge pattern.

Other systems are based on photometric stereo techniques [78,79], which estimate the three-dimensional shape of the ridges by evaluating the reflectance of the illuminated surface according to the position of the LEDs. Depth from focus methods [80] are also used to compute threedimensional models of the finger volume. Recent work has also examined the resonance-based analysis of A-scan signals for designing a multi-transducer acoustic imaging system for three-dimensional fingerprint imaging [81].

Three-dimensional models can be directly used by specifically designed feature extraction and matching techniques [83] or can be converted to touch-compatible fingerprint images using parametric or non-parametric unwrapping techniques.

The parametric method described in [84] approximates the finger's shape to a cylindrical model. The obtained images, however, exhibit horizontal distortions. To overcome this limitation, the method described in [74] approximates the finger's shape as a set of rings with different radii and center coordinates. The algorithm described in [85] approximates the finger as a sphere and then performs a linear mapping into a two-dimensional space by applying a distortion correction algorithm based on the analysis of the distances between adjacent points.

Some biometric systems $[70,71,76]$ use the non-parametric unwrapping technique described in [84]. This method aims to preserve the inter-point surface distances as much as possible. It divides the fingerprint model into slices along the vertical direction. A resampling algorithm applied to preserve the distances between the points pertaining to the slice then unfolds each slice. The non-parametric method presented in [86] fits the plane for each local region of the model and then unwraps the points for each fitted plane, minimizing a cost function that describes the movement exerted between each point and its neighbors. The method presented in [87] includes a simulation of the finger pressure on a sensor plate with the aim of increasing the similarity between touch-compatible images and touch-based images.

Techniques for simulating touchless fingerprint models $[88,89]$ and quality assessment methods for touchcompatible images obtained by unwrapping three-dimensional models [90] have also been reported. Recent studies have created synthetic three-dimensional fingerprint phantoms using three-dimensional printers [91], these phantoms may be particularly useful for designing both touch-based and touchless systems.

Commercial biometric systems based on three-dimensional touchless acquisition devices are also available. TBS 3D Terminal is based on a multiple-view acquisition method [92], while Flashscan 3D [93] uses a structured light technique. These systems use finger placement guides.

The literature also includes performance evaluations of commercial technologies for three-dimensional fingerprint reconstruction. The work presented in [94] evaluated the accuracy of the biometric recognition software Neurotechnology VeriFinger [95] using a dataset of 1,800 samples acquired from 150 individuals with the touchless device TBS S120 and a dataset of 1,800 touch-based images acquired from the same fingers with the touch-based sensor Crossmatch Verifier 300 [96]. The evaluated touchless device presented usability issues because it required many trials to acquire good-quality samples and it was not possible to collect samples from relatively short ring fingers and little fingers due to the shape of the acquisition device [94]. Moreover, the touchless acquisition technology presented a low level of interoperability with touch-based systems.

\section{The Proposed System}

The proposed system aims to obtain a recognition accuracy comparable to that of touch-based technologies while using a fully touchless acquisition procedure that is less-constrained, more usable, and more accepted by users than touch-based and three-dimensional fingerprint recognition systems in the literature. In particular, our system performs the acquisition automatically in a single instant of time as soon as the user's finger moves inside the field of view and focus region of the cameras.

The performed metric three-dimensional reconstruction is invariant to finger position. In fact, the three-dimensional 
reconstruction method computes the actual position of each point in the three-dimensional space (expressed in $\mathrm{mm}$ ). The size of the three-dimensional model only depends on the dimension of the real finger, thus avoiding possible problems due to scale variations typically present in two-dimensional acquisition systems. To compensate for possible rotations of the finger in the yaw, roll and pitch directions (Fig. 2), the proposed system includes a method that reduces finger placement differences between two acquisitions by simulating rotations of the three-dimensional finger shape.

In the enrollment phase, our system computes a threedimensional model and rotates the three-dimensional fingerprint $n_{R}$ times in three-dimensional space. Then, a "multitemplate" composed of $n_{R}$ two-dimensional templates is computed and stored in the database. In the verification and identification phases, the system creates a single template from the three-dimensional model of the finger. The identity comparison strategy consists of applying a fusion algorithm to the similarity scores obtained by matching the fresh template with all of the templates that compose the multi-template.

We use two-dimensional feature extraction and matching algorithms designed for touch-based images because they enable a demonstration of the compatibility and interoperability of the proposed system using current biometric recognition methods as well as direct comparison of the performance of the proposed system with that of touch-based technologies.

Fig. 3 outlines the biometric recognition process, which can be divided into the following steps:

1) acquisition,

2) three-dimensional fingerprint reconstruction,

3) computation of touch-compatible images,

4) template computation,

5) matching.

\section{A. Acquisition}

The acquisition setup is based on two calibrated CCD cameras $\left(\right.$ Camera $_{A}$ and Camera $\left._{B}\right)$ placed with an upward orientation. The calibration was performed off-line once using a chessboard captured in different positions. This task applies the algorithms described in $[97,98]$ to compute the intrinsic and extrinsic parameters of the two-view setup.

The acquisition setup does not use any finger placement guide, and the fingerprint is oriented downward, according to the most natural position of the hand. As soon as the moving finger is placed in the fields of view of the cameras, Camera $A$ and Camera $_{B}$ simultaneously capture the two-view images. We use a photocell and a trigger mechanism to capture images at the same instant of time, thus reducing possible variations of the finger position in the two-view images. The distance of the photocell from the cameras corresponds to the best focus plane.

The acquisition setup permits unconstrained finger placements with high rotations in the yaw, roll and pitch directions, as long as a sufficient area of the ridge pattern is visible in both captured images.

To enhance the visibility of the ridge pattern, we used a uniform green light. The light is triggered by the photocell

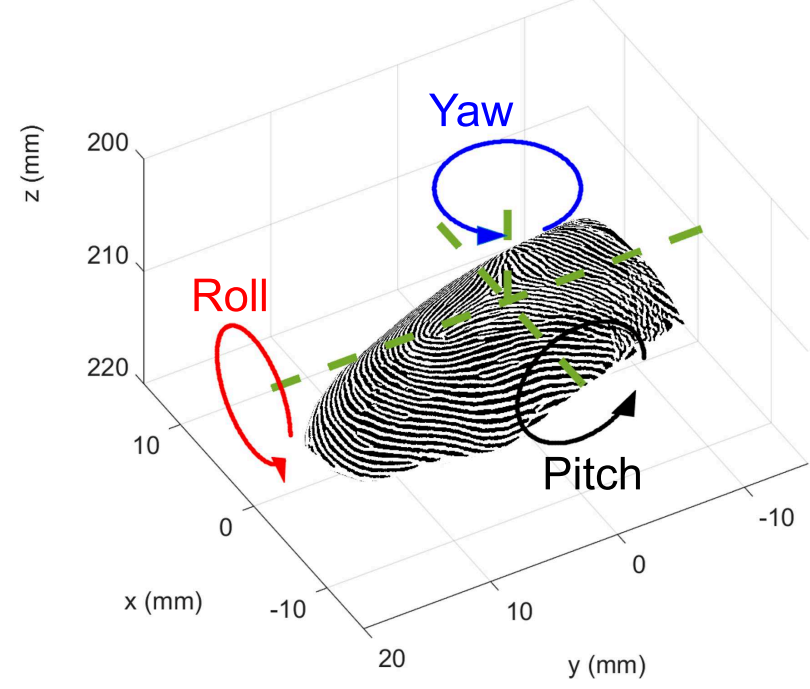

Fig. 2. Rotations of the finger with respect to the optical center of Camera $A$.

for a short time $t_{l}$, chosen to produce a light impulse almost invisible to the eye and able to reduce any blur effect due to movement of the user in front of the sensor. Moreover, the intensity of the light makes environmental illumination negligible. To simplify finger positioning, we projected a red cross light in the center of the acquisition area.

Fig. 4 presents examples of images captured by Camera $A_{A}$ and Camera $_{B}$.

\section{B. Three-dimensional fingerprint reconstruction}

The proposed method computes a three-dimensional model of the finger with a superimposed texture representing the ridge pattern. The method uses a correlation-based technique that is more computationally efficient and accurate than the one described in [36] and can be divided into the following tasks:

1) image preprocessing;

2) segmentation;

3) extraction and matching of the reference points;

4) refinement of the pairs of corresponding points;

5) three-dimensional surface computation and image wrapping.

1) Image preprocessing: Because a green light is used to illuminate the finger, the details of the ridge pattern are particularly visible in the green channel of the RGB color space of the captured images. Consequently, we consider the channel $G$ of the captured image $I$ (Fig. 5 a) as the matrix representing the ridge pattern $P$ (Fig. 5 b).

2) Segmentation: This task estimates the region of interest (ROI) of each image, which corresponds to the finger skin. The segmentation task also removes the fingernail from the ROI; the fingernail can cause deformations in the three-dimensional finger shape and introduce artifacts in the ridge pattern during subsequent computational steps.

The images are captured with the fingerprint oriented downward, and the background is the ceiling of the room. Thus, the background is out of focus due to its considerable distance 
(a)
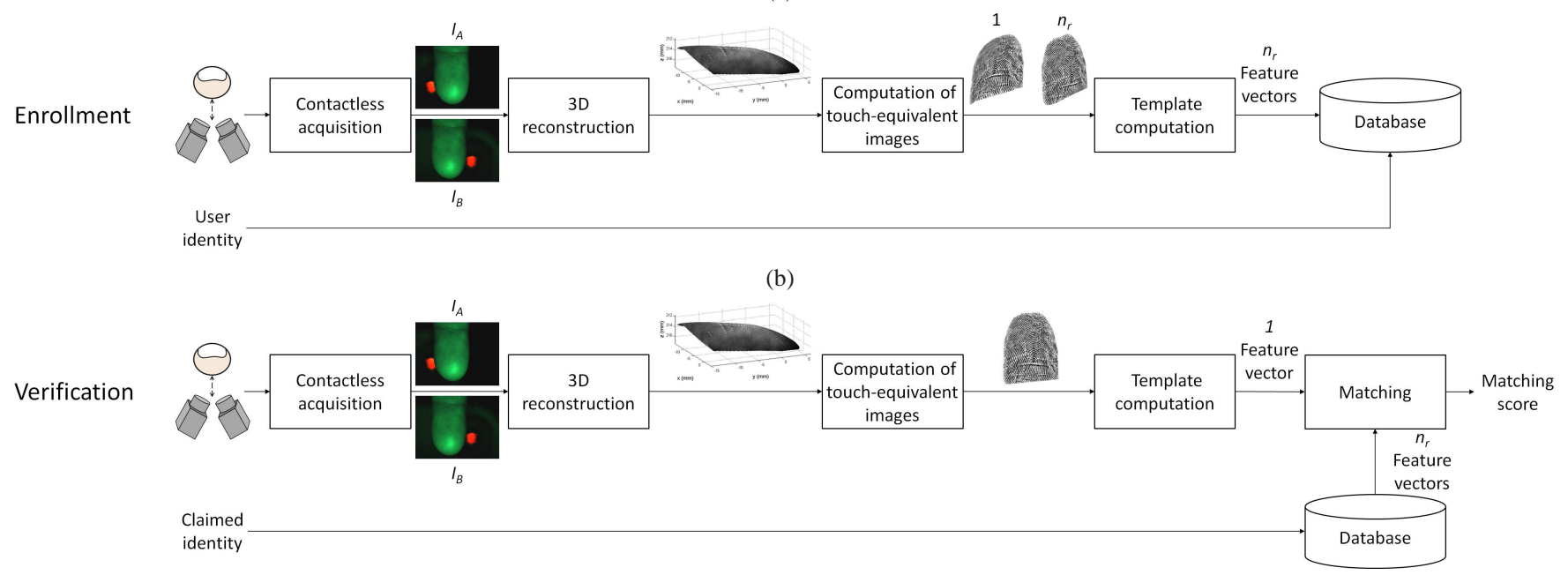

Fig. 3. Schema of the proposed system: (a) enrollment; (b) verification.

(a)

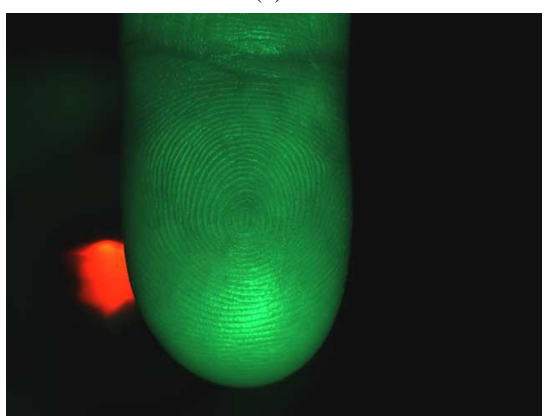

(b)

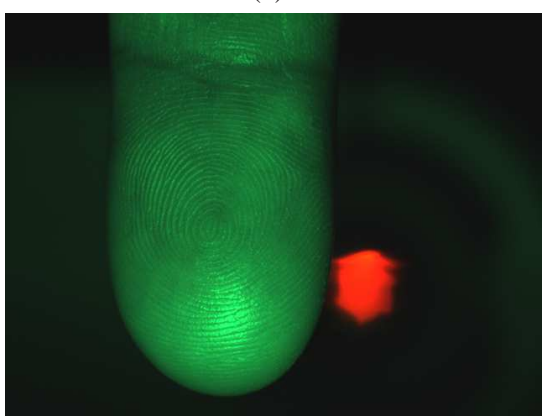

Fig. 4. Example of a two-view acquisition: (a) image $I_{A}$ captured by Camera $A$; (b) image $I_{B}$ captured by Camera ${ }_{B}$.

(a)

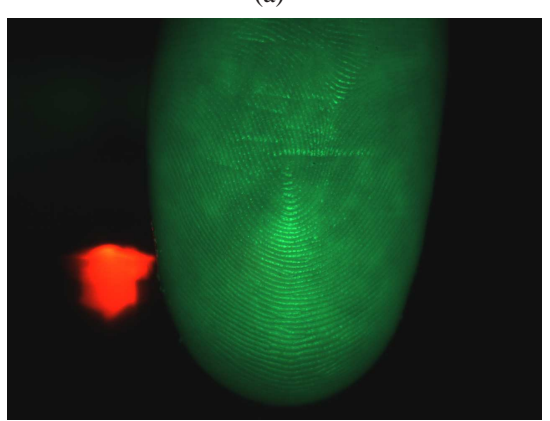

(b)

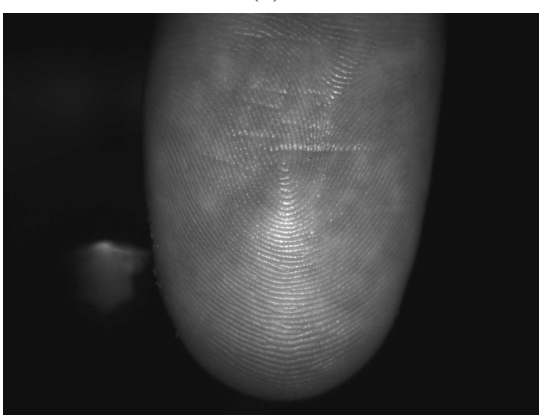

Fig. 5. Example of a fingerprint image before and after the preprocessing step: (a) input image $I$; (b) ridge pattern image $P$, computed as the green channel of $I$.

from the cameras and is mostly dark due to the large difference in radiation between the green LED light and the ambient light.

The segmentation task can be divided into two main tasks: rough estimation of the finger shape and fingernail removal. The last task estimates the fingernail as the image region exceeding a line perpendicular to the yaw angle of the finger and representing the termination of the skin region. Fig. 6 presents a schema of the overall segmentation process.

To estimate the finger shape, we use a simple thresholding technique. The binary image of the finger shape $B$ is computed from $P$ using a threshold $t_{o}$ that is empirically estimated for the dataset. $B$ is then refined using a morphological filling operator and eroded using a circular structural element with radius $r_{e}$ that is empirically estimated for the dataset.

The subsequent task is the removal of any potential fingernail regions from the segmented area. The proposed fingernail removal technique can be divided into the following tasks: compensation of the yaw angle, search for finger termination, and skin region segmentation.

In the first task, $P$ and $B$ are rotated by an angle of $-\alpha$ to make the finger's major axis vertical, resulting in the images $P_{-\alpha}$ and $B_{-\alpha}$, respectively. The variable $\alpha$ is computed as the 


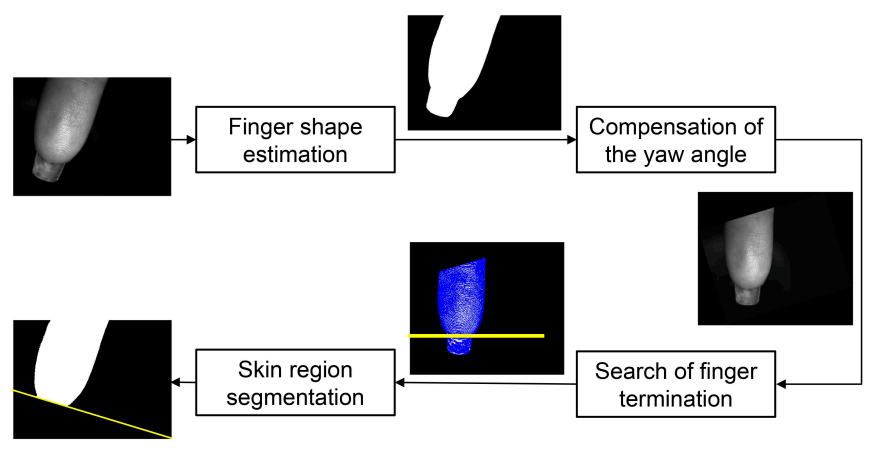

Fig. 6. Schema of the proposed segmentation method.

angle of an approximated line of symmetry $X_{c}(y)$ of $B$, which corresponds to the barycenter of the ROI of every coordinate $y$.

The estimation of $\alpha$ is performed as follows. For each $y$ coordinate of $B$, the points $X_{c}(y)$ are computed as the barycenter of the ROI:

$$
\left.X_{c}(y)=\underset{x=1 \ldots X}{\operatorname{argmin}}(B(x, y))+\underset{x=1 \ldots X}{\operatorname{argmax}}(B(x, y))\right) / 2,
$$

where $X$ is the size of $B$ in the $x$ direction. $\alpha$ is then estimated as the angle of the first-order polynomial approximating the vector $X_{c}$.

A line representing the finger termination is then assigned by evaluating features describing the presence of ridges and valleys. To find the $y$ coordinate corresponding to the finger's termination $y_{e}$, an image $G_{y}$ is then computed as the gradient of $P_{-\alpha}$ along the $y$ direction. A binary edge image $E_{y}$ is obtained from $G_{y}$ using threshold $t_{e}$, which is computed as the fixed $p_{e}$ percentile of $G_{y}$. The value of $p_{e}$ is empirically estimated for the used dataset.

The tomography of $E_{y}$ is subsequently computed. For each coordinate of the $y$ axis, $T(y)$ is obtained by the following:

$$
T(y)=\sum_{x=1}^{X} E_{y}(x, y) .
$$

A binary vector $B_{e}$, describing the presence of ridges, is computed from $T(y)$ by using the threshold $t_{r}$. This parameter has been empirically estimated for the used dataset.

The $y$ coordinate $y_{e}$ of the line corresponding to the end of the finger's skin is then estimated by the following:

$$
y_{e}=\underset{y=1 \ldots Y}{\operatorname{argmax}}(T(y)),
$$

where $Y$ is the size by $B_{-\alpha}$ in the $y$ direction.

The skin region segmentation is finally performed by setting the points of $B_{-\alpha}$ below $y_{e}$ to 0 , and the final ROI $B_{f}$ is obtained by rotating $B_{-\alpha}$ of $\alpha$ degrees.

3) Extraction and matching of the reference points: To reduce both the computational complexity and the likelihood of false matches, the image $P_{A}$ (captured by the Camera ${ }_{A}$ ) is first rectified such that corresponding epipolar lines of $P_{B}$ (captured by the $\mathrm{Camera}_{B}$ ) lie along horizontal scan-lines [99]. This task produces the image $P_{A}^{\prime}$ and is performed using the homography matrix $H$ obtained from the calibration step.
Next, a set of regularly spaced points $X_{A}^{\prime}$ is selected by downsampling the ROI of $P_{A}^{\prime}$ with a step of $s_{d}$ pixels. In the following, we refer to these points as reference points.

For each $x_{A}^{\prime} \in X_{A}^{\prime}$, the corresponding point $x_{B}$ in $P_{B}$ is estimated using a block matching technique. The method searches for the best normalized cross-correlation between a $l \times l$ squared window centered in the coordinates $x_{A}^{\prime}$ of the image $P_{A}^{\prime}$, and a $l \times l$ squared window sliding in the corresponding epipolar line of $P_{B}$. We searched corresponding points only in the horizontal direction because the image rectification made the pairs of conjugate epipolar lines of the two-view images collinear and parallel to the $y$ axis. The search range is limited to $\pm w$ pixels along the $x$ axis. For each reference point of the image $P_{A}^{\prime}$, the corresponding point $x_{B}$ of $P_{B}$ is considered as the pixel with the best correlation value in the search range of the second view.

Finally, the coordinates of the matched points $x_{A}^{\prime}$ are mapped into the space of the image $P_{A}$ as follows:

$$
x_{A}=H^{T} x_{A}^{\prime} .
$$

The studied acquisition setup achieves a good maximization of the small local variations of finger skin characteristics, allowing the matching algorithm to identify a large number of robust pairs of corresponding points.

Fig. 7 depicts a visual representation of the proposed algorithm for estimating corresponding pairs of points. Fig. 8 shows portions of two-view images and the computed pairs of corresponding points.

4) Refinement of the pairs of corresponding points: To obtain a smooth and accurate representation of the finger surface, we applied a thin plate spline to the set of corresponding points. Fig. 9 presents examples of point clouds obtained with and without the refinement step.

5) Three-dimensional surface computation and image wrapping: This task creates a three-dimensional model $M_{A}$ as the depth map corresponding to the view of Camera ${ }_{A}$ by computing the three-dimensional shape of the finger $M_{z}$ and wrapping an image of the ridge pattern $M_{p}$ on the estimated model.

The three-dimensional reconstruction method computes the depth coordinate of every three-dimensional point using the following triangulation formula:

$$
z=\frac{f T}{x_{A}-x_{B}},
$$

where $f$ is the focal length of the two cameras, $T$ is the baseline distance between the two cameras, and $x_{A}$ and $x_{B}$ are the two matched points.

We compute $M_{z}$ and $M_{p}$ from the obtained point cloud using an interpolation approach. Two matrices $M_{x}$ and $M_{y}$, representing the $x$ and $y$ coordinates of the three-dimensional model, are computed as equally spaced grids with a sampling step equal to $s_{i}$. The value $s_{i}$ is empirically estimated with the aim of obtaining a good tradeoff between the computational efficiency and quality of the three-dimensional models. $M_{z}$ is obtained by a linear interpolation of the estimated $z$ values in the coordinates described by $M_{x}$ and $M_{y} . M_{p}$ and the binary map of the ROI $M_{b}$ are obtained by applying the 
(a)

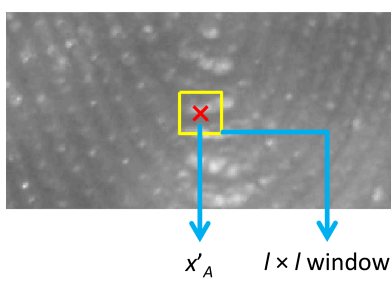

(b)

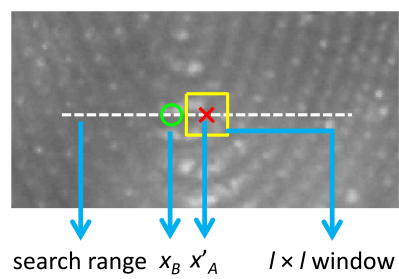

(c)

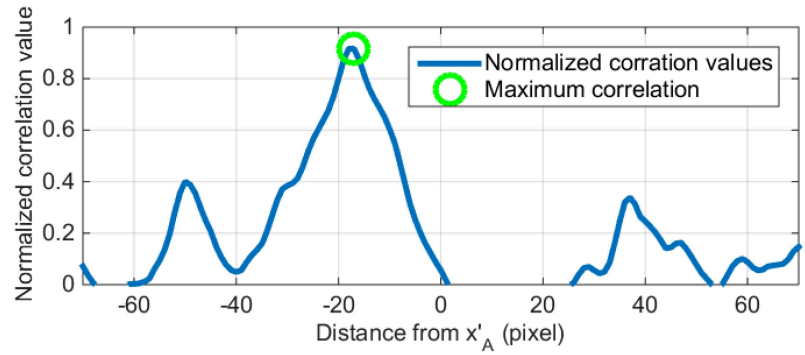

Fig. 7. Representation of the proposed algorithm for estimating corresponding pairs of points: (a) selected point $x_{A}^{\prime}$ in the rectified image $P_{A}^{\prime}$ and boundaries of the corresponding $l \times l$ local region; (b) $x_{A}^{\prime}$ in the image $P_{B}$, boundaries of the corresponding $l \times l$ local region, search region of $\pm w$ pixels along the $x$ axis (corresponding to an epipolar line of $P_{A}$ ), and matched point $x_{B}$; (c) correlation values in the search region. Because the ridge pattern is highly repetitive, we studied the illumination system and the characteristics of the cameras used by the acquisition setup to maximize the small local variations of the finger skin characteristics. The search of corresponding points in the acquired images can therefore obtain high differences in the normalized correlation values, and properly match the great majority of the considered points.

same interpolation technique to the points of $P_{A}$ and $B_{f}$, respectively.

Fig. 10 shows an example of a dense three-dimensional model with a superimposed texture image.

\section{Computation of touch-compatible images}

Starting from a three-dimensional model of the finger surface, the proposed method computes touch-compatible images that represent the ridge pattern of acquisitions performed with arbitrary finger rotations. We refer to these images as touchcompatible because we have experimentally demonstrated that these images can be processed using algorithms designed for touch-based images.

The results are gray-scale images with a standard resolution of 500 DPI. Images with a standard fixed resolution can be obtained because three-dimensional models are computed by performing a metric reconstruction. Therefore, the size of each three-dimensional model corresponds to the real size of the corresponding finger (expressed in $\mathrm{mm}$ ).

The method can be divided into the following tasks:

- enhancement;

- two-dimensional mapping.

1) Enhancement: The method computes an enhanced texture $M_{e}$ from $M_{p}$ using algorithms similar to those presented in [25].

A background image $I_{b}$ is estimated by applying a morphological opening operation (with a structuring element of $s_{e}$ pixels) to $M_{p}$. Then, the background is subtracted from $M_{p}$, resulting in $I_{r}$. (a)

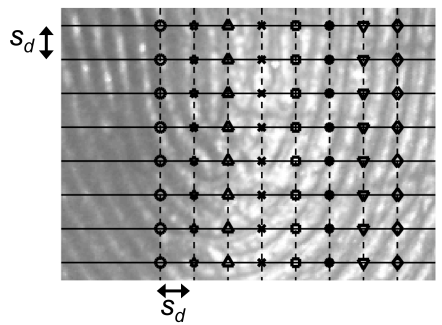

(b)

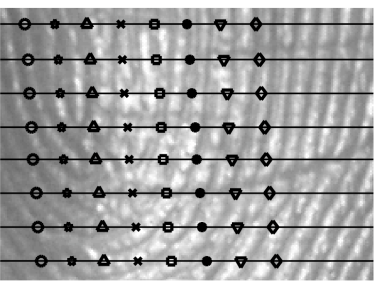

Fig. 8. Portions of two-view images and the corresponding matched points: (a) portion of the rectified image $P_{A}^{\prime}$; (b) portion of the image $P_{B}$. The images are cropped starting from the same coordinates. The horizontal lines correspond to epipolar lines. The horizontal lines and vertical dashed lines in image (a) represent the used sampling strategy and are separated by $s_{d}$ pixels. Markers lying on the same epipolar lines represent matched pairs of points. The proposed matching method overcomes illumination differences and perspective deformations due to the placement of the cameras, accurately estimating pairs of corresponding points in the two-view images.

To increase the visibility of the ridge pattern, a nonlinear equalization is performed as $I_{l}(x, y)=\log \left(I_{r}(x, y)\right)$.

An 8-order Butterworth low-pass filter [100] with a frequency $f_{f}$ and size $d_{f} \times d_{f}$ is then used to reduce noise. We have selected a Butterworth filter instead of other types of low-pass filters because it experimentally obtained sharper images with higher contrast between ridges and valleys. The values of $f_{f}$ and $d_{f}$ were then empirically estimated.

The enhanced texture $M_{e}$ is finally obtained by applying a histogram equalization to $I_{l}$.

2) Two-dimensional mapping: This task computes $n_{R}$ touch-compatible images from a single three-dimensional model of the finger surface. It compensates for the acquisition angle along the yaw direction and simulates $n_{R}$ acquisitions performed with known rotation angles in the roll and pitch directions. In the enrollment phase, a value of $n_{R}$ equal to or greater than 1 is applied to compensate for possible misplacements of the finger during future acquisitions of probe samples. In the verification and identification phases, we compute a single touch-compatible image $\left(n_{R}=1\right)$ from the fresh sample.

To obtain the same number of roll and pitch rotations, including the null rotation, the possible values of $n_{R}$ are chosen as squares of integer odd numbers.

To compensate the finger placement angle along the yaw direction $\phi$, we apply the same algorithm used to remove the finger nail to $M_{b}$ and then compute the rotation matrix $R_{x}$, which is obtained as follows:

$$
R_{x}(\phi)=\left[\begin{array}{ccc}
1 & 0 & 0 \\
0 & \cos (\phi) & \sin (\phi) \\
0 & -\sin (\phi) & \cos (\phi)
\end{array}\right]
$$

Then, a set of $\sqrt{n_{R}}$ equally spaced roll angles $\Theta(i)$ and the corresponding rotation matrices $R_{y}(\Theta(i))$ are computed as follows: 
(a)

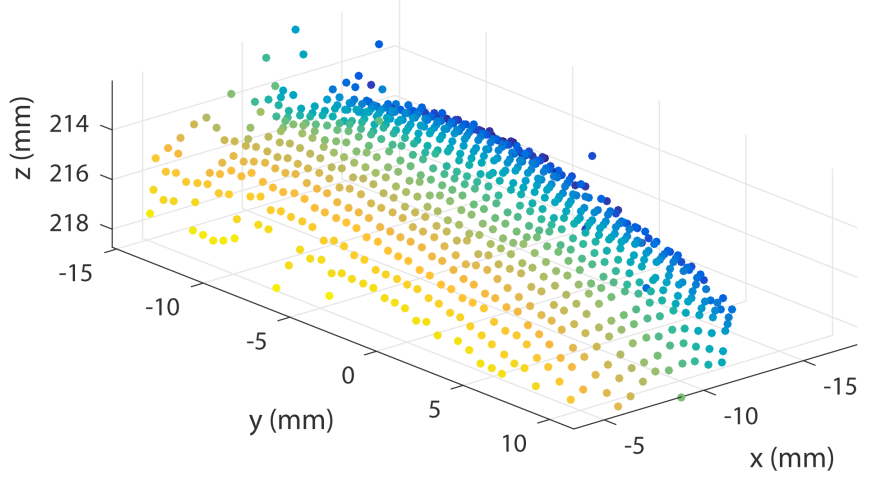

(b)

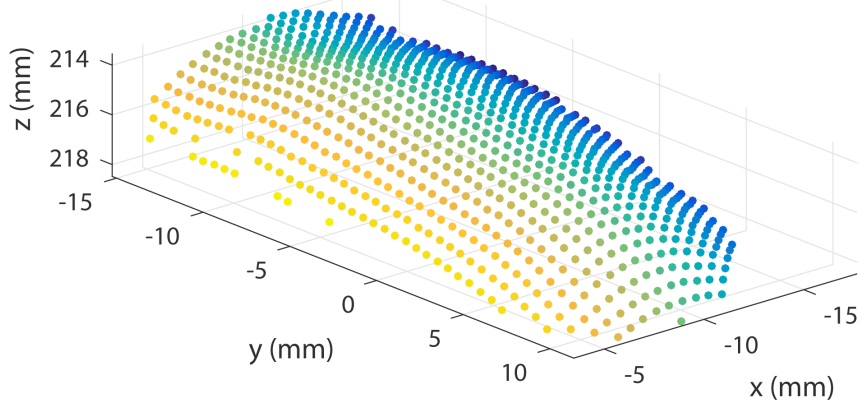

Fig. 9. Application of the method for refining the pairs of corresponding points: (a) an example of an input point cloud; (b) application of the refinement step.

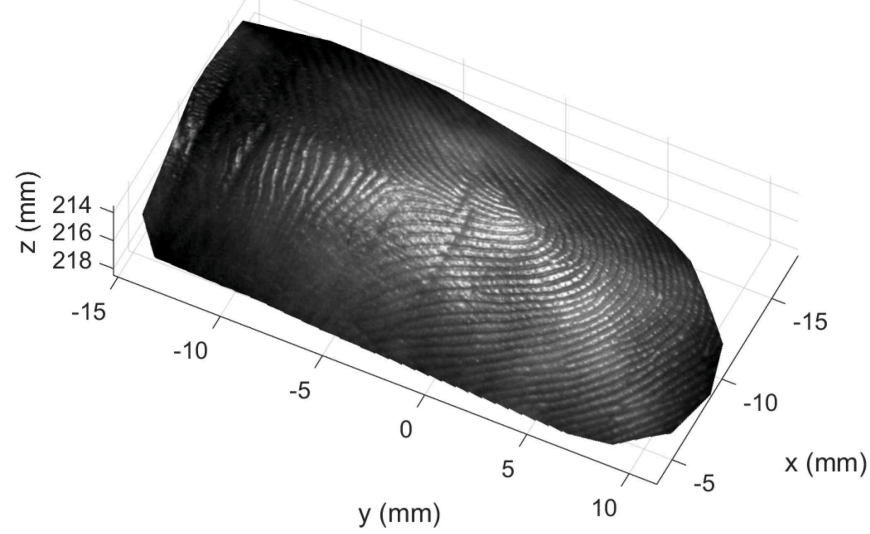

Fig. 10. Example of a dense three-dimensional model with a superimposed texture image obtained by the proposed method.

$$
\begin{array}{r}
\Theta(i)=i \Delta_{R} \\
i=-\left\lfloor\left(\left(\sqrt{n_{R}}\right) / 2\right)\right\rfloor \ldots+\left\lfloor\left(\left(\sqrt{n_{R}}\right) / 2\right)\right\rfloor, i \in Z, \\
R_{y}(\Theta(i))=\left[\begin{array}{ccc}
\cos (\Theta(i)) & 0 & -\sin (\Theta(i)) \\
0 & 1 & 0 \\
\sin (\Theta(i)) & 0 & \cos (\Theta(i))
\end{array}\right],
\end{array}
$$

where $\Delta_{R}$ is the constant angle rotation step and $\lfloor\cdot\rfloor$ is the floor operator.

In a similar manner, a set of $\sqrt{n_{R}}$ equally spaced pitch angles $\Psi(j)$ and the corresponding rotation matrices $R_{z}(\Psi(j))$ are computed as follows:

$$
\begin{array}{r}
\Psi(j)=j \Delta_{R} \\
j=-\left\lfloor\left(\left(\sqrt{n_{R}}\right) / 2\right)\right\rfloor \ldots+\left\lfloor\left(\left(\sqrt{n_{R}}\right) / 2\right)\right\rfloor, j \in Z, \\
R_{z}(\Psi(i))=\left[\begin{array}{ccc}
\cos (\Psi(j)) & \sin (\Psi(j)) & 0 \\
-\sin (\Psi(j)) & \cos (\Psi(j)) & 0 \\
0 & 0 & 1
\end{array}\right] .
\end{array}
$$

For each roll angle $\Theta(i)$ and pitch angle $\Psi(i)$, we rotate the coordinates $M_{x}, M_{y}$ and $M_{z}$ of the three-dimensional model $M_{A}$ in the three-dimensional space with a rotation matrix $R(\Theta(i), \Psi(j))$, which is obtained as follows:

$$
R(\Theta(i), \Psi(j))=R_{x} R_{y}(\Theta(i)) R_{z}(\Psi(j)) .
$$

Every rotated touch-compatible image $E_{(\Theta(i), \Psi(j))}$ is obtained by applying a re-sampling with a constant step equal of $1 / 500$ inch to the enhanced texture image $M_{e}$ in the new $x$, $y$ and $z$ coordinates. This computation is based on a bilinear interpolation.

To remove regions of the images that do not pertain to the last phalanx, we crop the touch-compatible images at a fixed length of $l_{e} \mathrm{~mm}$ from the finger end in the $y$ direction.

Fig. 11 presents examples of touch-compatible images obtained from the three-dimensional model shown in Fig. 10, and computed with an angular step $\Delta_{R}=15^{\circ}$. As shown in Fig. 11, small rotations in the three-dimensional space produce substantial changes in the touch-compatible images.

\section{Template computation}

To demonstrate the full compatibility with standard biometric recognition technologies for fingerprint recognition, we have adopted well-known methods designed for touch-based images. Minutiae templates $T$ are computed using the software Neurotechnology VeriFinger [95]. As an example, Fig. 12 shows a binarized touch-compatible image and the extracted minutia coordinates.

\section{E. Matching}

The enrollment phase creates a multi-template composed by $n_{R}$ minutiae templates $T_{e}$ from $n_{R}$ touch-compatible images obtained by applying the proposed two-dimensional mapping technique. The verification phase computes a single minutiae template $T_{f}$ from the touch-compatible image $E_{\left(0^{\circ}, 0^{\circ}\right)}$.

The matching score represents the similarity of the fresh template with a multi-template. This score is computed as follows:

$$
\text { matching score }=\max _{i=1 \ldots n_{R}}\left(\operatorname{match}\left(T_{e}(i), T_{f}\right)\right),
$$

where match $(\cdot)$ represents the matching function, which is performed using Neurotechnology VeriFinger software. 
(a)

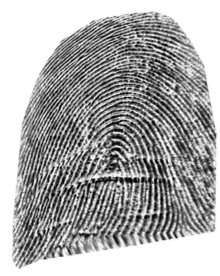

(d)

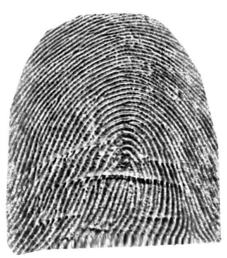

(g)

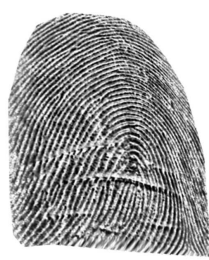

(b)

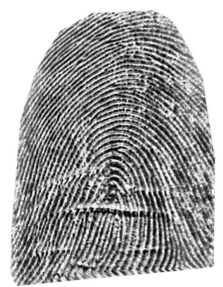

(e)

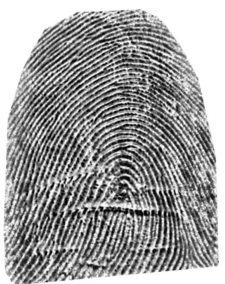

(h)

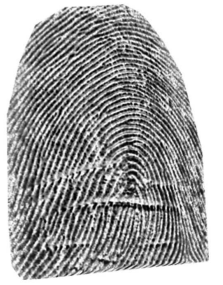

(c)

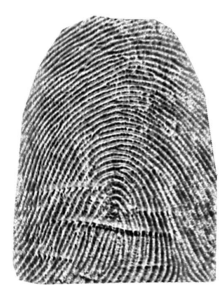

(f)

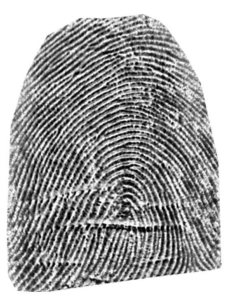

(i)

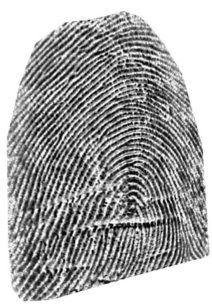

Fig. 11. Examples of touch-compatible gray-scale images $E_{(\Theta, \Psi)}$ obtained by the proposed system with $n_{R}=9$ and $\Delta_{R}=15^{\circ}:$ (a) $E_{\left(-15^{\circ},-15^{\circ}\right)}$; (b) $E_{\left(0^{\circ},-15^{\circ}\right)}$; (c) $E_{\left(15^{\circ},-15^{\circ}\right)}$; (d) $E_{\left(-15^{\circ}, 0^{\circ}\right)}$; (e) $E_{\left(0^{\circ}, 0^{\circ}\right)}$; (f) $E_{\left(15^{\circ}, 0^{\circ}\right)}$; (g) $E_{\left(-15^{\circ}, 15^{\circ}\right)}$; (h) $E_{\left(0^{\circ}, 15^{\circ}\right)}$; (i) $E_{\left(15^{\circ}, 15^{\circ}\right)}$. Small rotations in the threedimensional space produce large changes in the touch-compatible images. In contrast to other methods presented in the literature in which these differences are critical, the proposed is unaffected by these differences.

\section{EXPERIMENTAL Results}

The performed experiments aim to evaluate different characteristics of the proposed biometric system by performing technology and scenario evaluations [101]. We performed an analysis of the accuracy of the proposed three-dimensional reconstruction method using both quantitative and qualitative measurements $[1,102]$. Next, we evaluated the performance of the biometric system on a dataset of more than 2,300 samples. We also conducted tests to evaluate the robustness of the system to different environmental light conditions, its sensitivity, its robustness to intentional misplacements of the finger during the acquisition process, and the computational time required by the proposed algorithms. Then, the resulting figures of merit were compared with those for traditional touch-based technologies and the most accurate touchless fingerprint recognition systems in the literature. We also evaluated the performance of the proposed system for data acquisition over one year. Moreover, we examined the compatibility and interoperability of the proposed system with touch-based samples. Finally, we evaluated user acceptability by analyzing the results of evaluation forms completed by volunteers after using both the proposed touchless system and touch-based sensors.

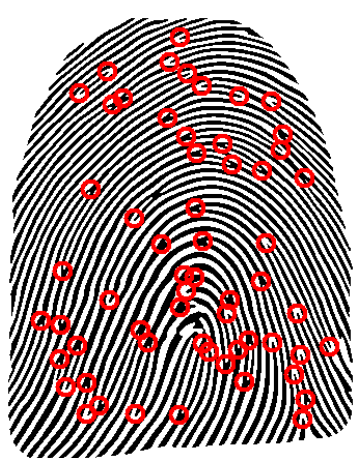

Fig. 12. Example of a binary image and minutiae coordinates obtained using the proposed system and the software Verifinger. Notably, this commercial feature extractor properly identified the coordinates of the minutiae obtained by the proposed touchless method.

\section{A. Experimental design}

In the following, we describe the experimental setup, the parameters used by the proposed algorithms, and the collected datasets.

1) Acquisition setup: Fig. 13 a presents the setup configuration. Acquisitions were performed at a mean distance $\Delta_{H}=220 \mathrm{~mm}$ between the finger and the CCD of every camera. The angle of the cameras with respect to the horizontal support was $\theta=85^{\circ}$, with a baseline distance between the cameras $\Delta_{D}=65 \mathrm{~mm}$ (from the centers of the CCDs). The light source was placed at a distance of $\Delta_{L}=150 \mathrm{~mm}$.

The cameras captured images at a resolution of $1280 \times$ 960 pixels. The optics were Tamron $20 \mathrm{HC}$ with a $25-\mathrm{mm}$ focal length. Images were captured with aperture $F=3$, a focal length of $0.25 \mathrm{~m}$, a shutter time of $529 \mu \mathrm{s}$, and an acquisition gain of 100. The light impulse had a duration $t_{l}=500 \mu \mathrm{s}$. The illumination system consisted of an LED lamp with 7 contiguous green light sources.

The real acquisition system was covered by a box (Fig. $13 \mathrm{~b}$ ) to protect the hardware components, simplify the search of the acquisition area during the acquisition process, and provide a simple prototype of a possible industrial implementation of the system.

2) Parameters of the implemented algorithms: To achieve correct behavior under the largest range of operative conditions, the parameters of the implemented methods were empirically focused on the separate dataset Dataset $_{\text {Calibration }}$ of samples representing very different acquisitions in term of finger positioning. The parameters of the segmentation step were $t_{o}=45, r_{e}=20$ pixels, $p_{e}=80 \%, t_{r}=0.1$. The parameters used to compute three-dimensional models were $s_{d}=20$ pixels, $l=21$ pixels, $w=70$ pixels. The maximum length of the cropped touch-compatible images was $l_{e}=230 \mathrm{~mm}$. The sampling step used for computing the dense three-dimensional models was $s_{i}=0.025 \mathrm{~mm}$, corresponding to a spatial resolution of approximately 1000 PPI. The parameters of the image enhancement method were $s_{E}=15$, $d_{f}=20$, and $f_{f}=0.1$.

3) Created datasets: To analyze the robustness of the system to different environmental light conditions and intentional finger misplacement, the performance of the proposed 
(a)

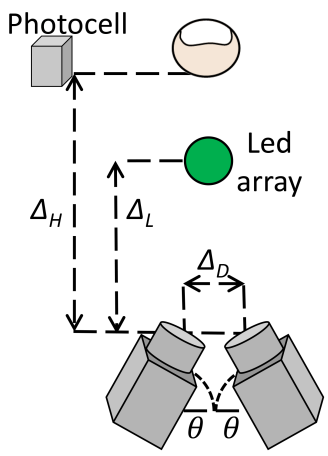

(b)

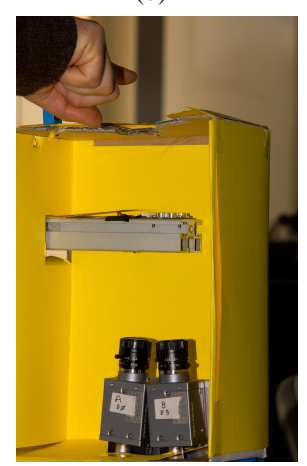

Fig. 13. The acquisition setup: (a) schema of the acquisition setup; (b) photograph of the setup and finger positioning. We covered the acquisition system with a box to protect the hardware components and simplify the search of the acquisition area during the acquisition process.

biometric system was evaluated using different datasets created in our laboratory. As a reference, we collected touch-based samples from the same volunteers who provided touchless samples.

Biometric data were collected from a set of 30 volunteers including both men and women. The volunteers included graduate students, workers, and retirees ranging in age from 20 to 64 years old. We acquired data during two sessions performed over the course of a year. A trained operator supervised the acquisition of both touch-based and touchless samples. The hardware setup was disassembled after the first session and then assembled again to perform the second acquisition session. For each session, a setup calibration was performed. The acquisition procedure required very little training of the volunteers: they were only trained on where to place the finger to be acquired by both of the cameras. A description of every dataset is reported in the following.

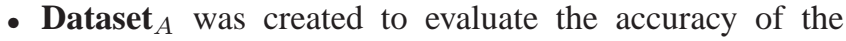
implemented touchless recognition system for samples acquired with standard finger placements under different light conditions. We performed the acquisitions at different hours of the day (from 9 am to $8 \mathrm{pm}$ ) both with and without artificial illumination of the room. The dataset was composed of 2,368 samples acquired in a single session. Images of the ten fingers of 30 volunteers were collected. Each finger was imaged 8 times ( 4 times with artificial environmental light and 4 under natural light conditions) to obtain a total of 80 images for each volunteer. It was not possible to capture samples from 4 fingers because of cuts or amputations. Fig. 14 presents examples of images pertaining to Dataset $_{A}$.

- Dataset ${ }_{B}$ was created to evaluate the robustness of the implemented touchless recognition system to intentional misplacements and finger rotations. Because it is likely impossible to place a finger with a known angle without using finger guides, we asked the users to place the finger with high rotations (approximately $30^{\circ}$ ) in the yaw, roll, and pitch directions. Users considered the requested finger rotations exaggerated with respect to the natural position of the finger on the proposed acquisition system.
Most of the samples were intentionally misplaced to be reasonably considered as failures to acquire (FTA) in commercial touch-based scanners. The dataset was composed of 1,200 samples acquired in a single session. The two index fingers of each volunteer were captured 8 times without asking to perform rotations and 2 times for each of the 6 considering orientation: leftward yaw rotation, rightward yaw rotation, counterclockwise roll rotation, clockwise roll rotation, downward pitch rotation, upward pitch rotation. A total of 20 acquisitions for each index finger and 40 acquisitions for each volunteer were performed. A visual analysis of the samples captured asking for finger rotations indicated a variability of the orientation from approximately $20^{\circ}$ to approximately $45^{\circ}$. Fig. 15 presents examples of images captured with different finger placements. The exaggerated rotations of the samples in Dataset ${ }_{B}$ are apparent.

- Dataset D $_{T}$ was created as a reference touch-based dataset to compare the implemented touchless system with stateof-the-art touch-based technologies. The dataset was composed of 1,184 images acquired in a single session using the touch-based sensor Crossmatch Verifier 300 [96]. The ten fingers of each volunteer were captured 4 times. It was impossible to capture samples of 4 of the fingers due to cuts or amputations.

- Dataset DY $_{A}$ was created to evaluate the accuracy of the system over a time interval of one year. The dataset was composed of 2,368 samples acquired in two sessions. Images from ten fingers of 15 volunteers were collected. Each finger was imaged 16 times ( 8 samples in Dataset $A$ and 8 samples acquired in the second session) to obtain a total of 160 images for each volunteer. It was impossible to capture samples of 2 of the fingers because of cuts or amputations.

- Dataset $_{T Y}$ was created as a reference touch-based dataset to compare the performance of touch-based and touchless technologies over a time interval of one year. The dataset was composed of 1,184 images acquired during two sessions using a Crossmatch Verifier 300. For each volunteer, the ten fingers were captured 8 times ( 4 samples in Dataset $_{T}$ and 4 samples acquired in the second session). It was impossible to capture samples of 2 of the fingers due to cuts or amputations.

\section{B. Evaluation of the three-dimensional reconstruction}

We performed both visual and numerical analyses to evaluate the accuracy of the proposed three-dimensional reconstruction method. The results confirmed that the proposed less-constrained acquisition technique and three-dimensional reconstruction method achieved sufficient accuracy for use in biometric systems. Fig. 16 presents examples of reconstructed three-dimensional point clouds, the corresponding interpolated surfaces, and superimposed texture images. The three-dimensional reconstruction method effectively modeled the finger's shape. We evaluated the accuracy of the system calibration using the method described in [103] and obtained an error of $0.03 \mathrm{~mm}$. This method computes the threedimensional reconstruction error by triangulating the corner 
(a)

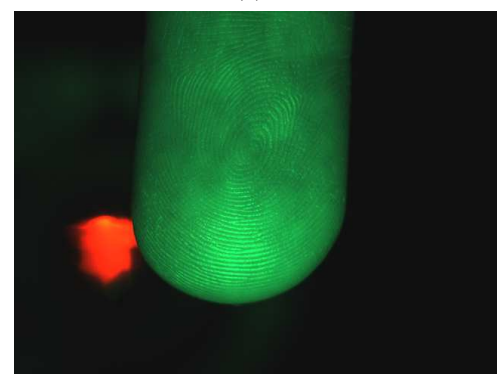

(d)

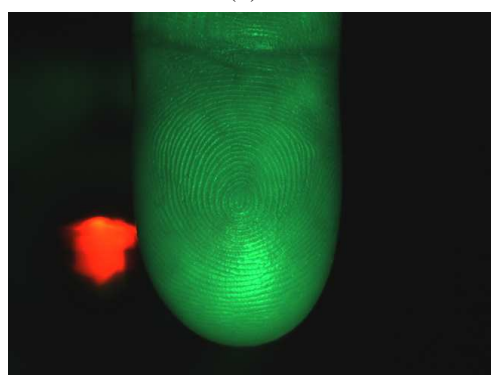

(b)

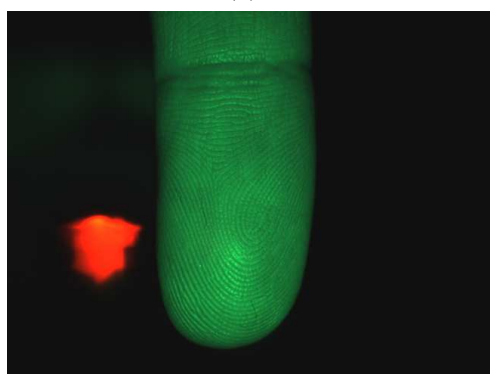

(e)

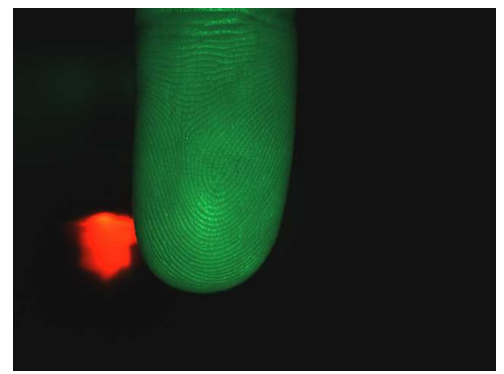

(c)

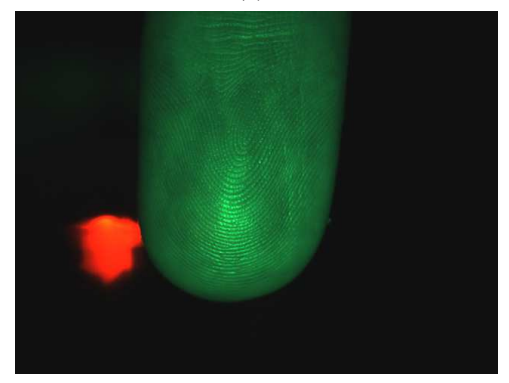

(f)

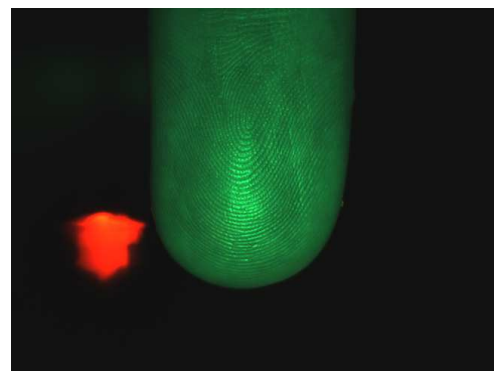

Fig. 14. Examples of acquisitions of Dataset $A$ performed with and without artificial illumination of the room: (a-c) fingers captured without artificial illumination; (d-f) the same fingers captured with artificial illumination. The light used to enhance the visibility of the ridge pattern is sufficiently intense to make the environmental illumination negligible. Moreover, the impulse duration is sufficiently short to be nearly invisible to the eye and to reduce any blur effects due to the movements of the user in front of the sensor.

(a)

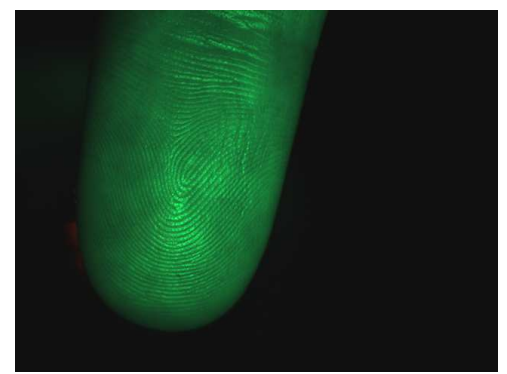

(d)

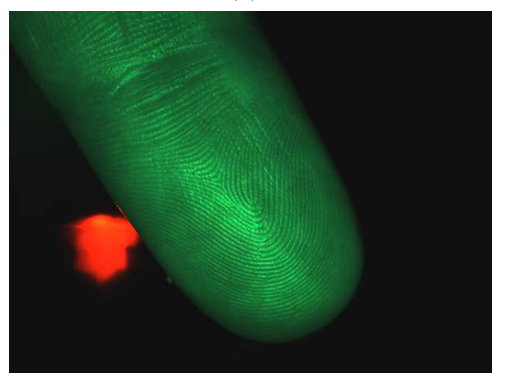

(b)

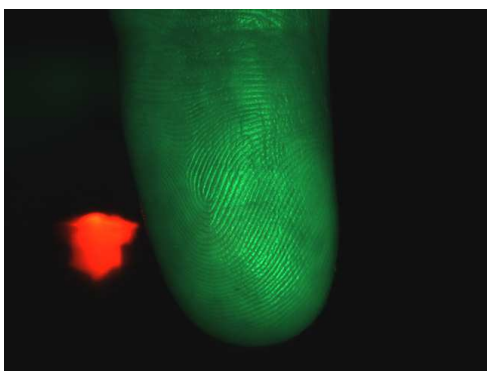

(e)

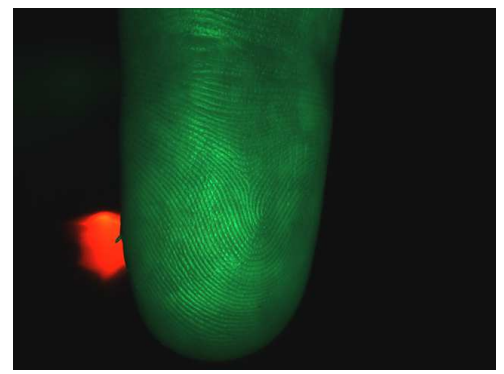

(c)

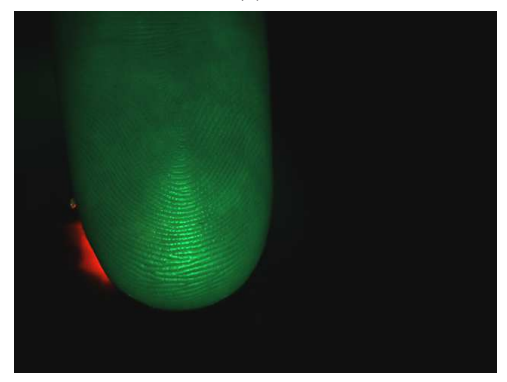

(f)

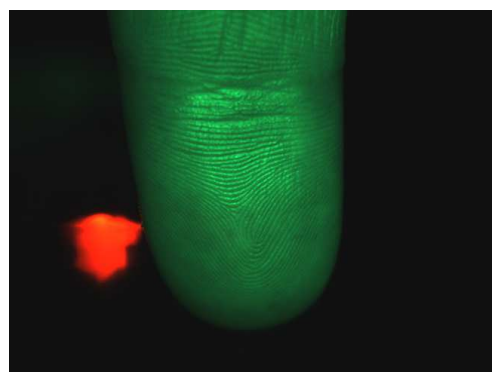

Fig. 15. Examples of images in Dataset . $_{B}$ captured with exaggerate finger orientations: (a) leftward yaw rotation; (b) counterclockwise roll rotation; (c) downward pitch rotation; (d) rightward yaw rotation; (e) clockwise roll rotation; (f) upward pitch rotation.

coordinates of the chessboard images used to calibrate the system and by interpolating a plane passing from the obtained three-dimensional coordinates. The final result is the standard deviation of the Euclidean distance between the threedimensional corners and the three-dimensional plane.

We also examined the accuracy of the algorithm used to search the corresponding points in the two-view images. The performed test included computing the absolute distance along the $z$ axis between the three-dimensional points obtained before and after the noise reduction step by assuming the continuity of the finger surface. A mean error of $0.17 \mathrm{~mm}$ was obtained. This value is satisfactory because three-dimensional models obtained by multiple-view systems typically present outliers.

The number of corresponding points in the input images is due to the sampling step $s_{d}$. We observed $s_{d}=20$ pixels was a good tradeoff between three-dimensional reconstruction quality and required computational time. The mean number of 
(a)

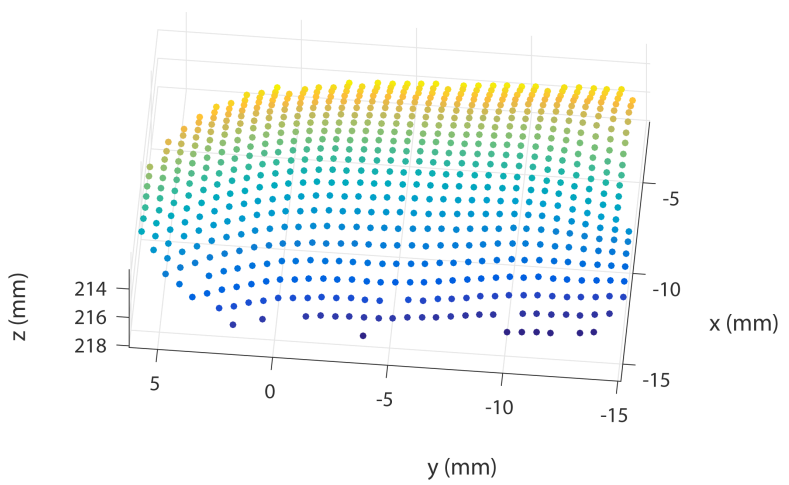

(c)

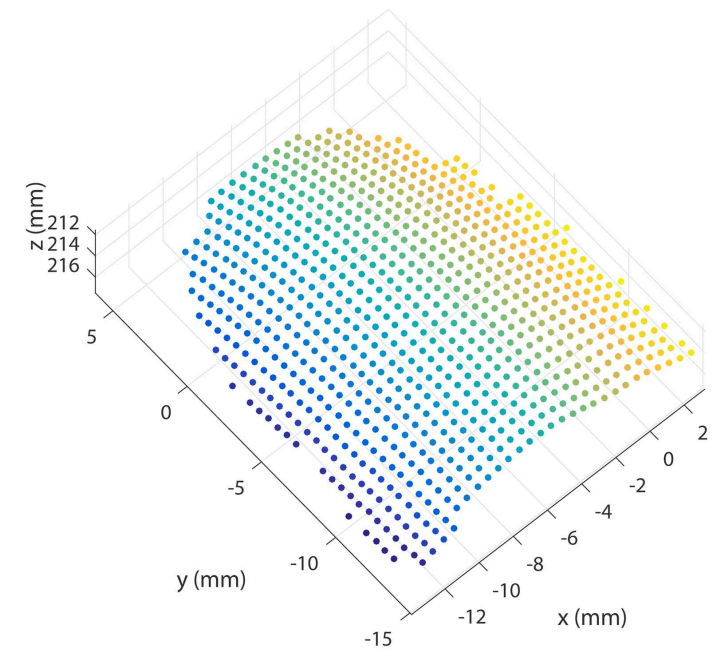

(e)

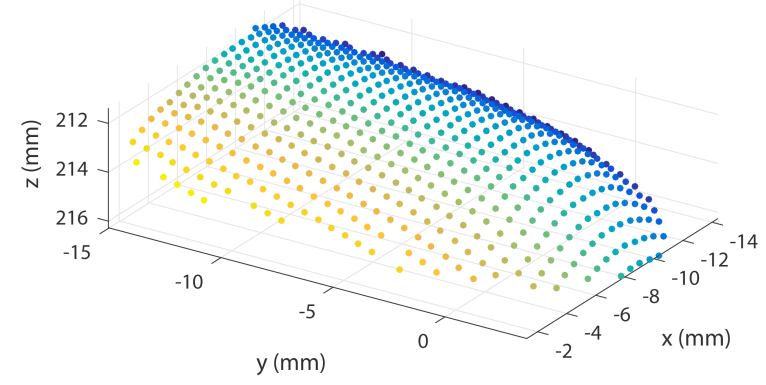

(b)

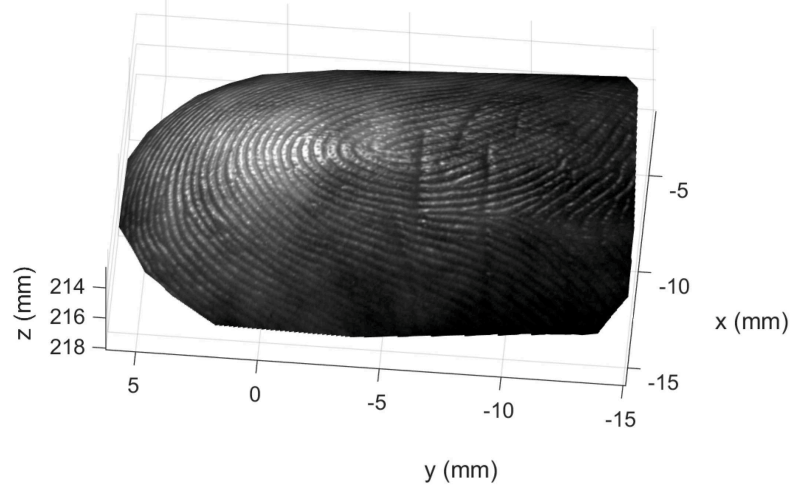

(d)

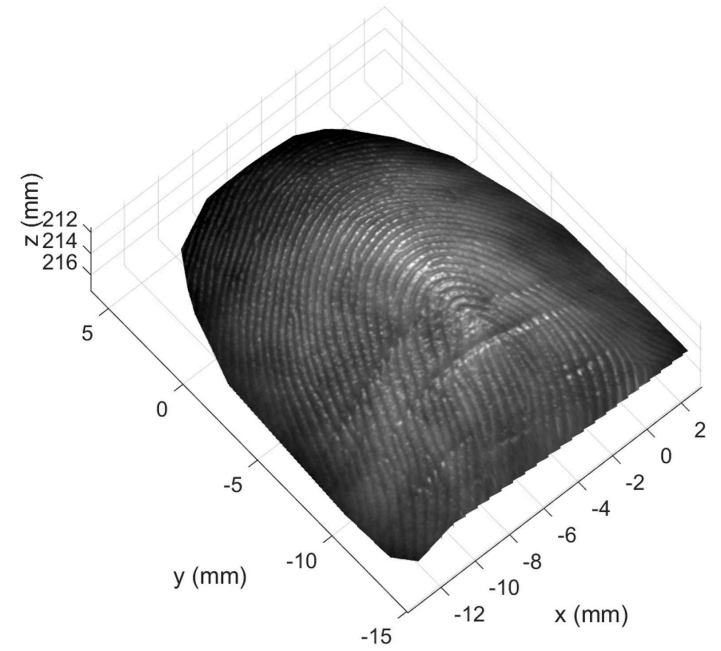

(f)

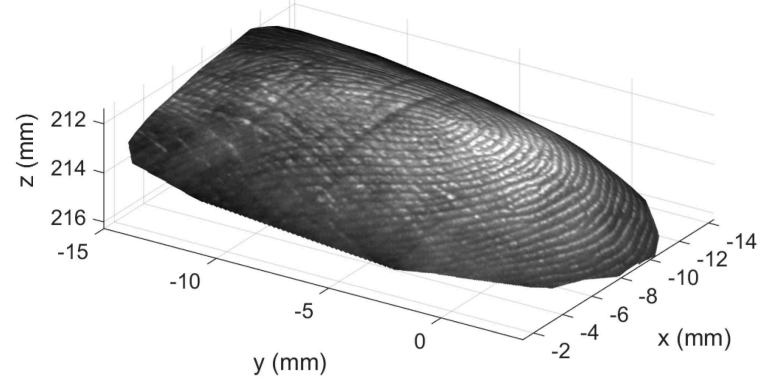

Fig. 16. Examples of point clouds describing the three-dimensional shape of the finger and the corresponding dense three-dimensional models with superimposed texture images: (a, c, e) point clouds; (b, d, f) dense three-dimensional models. The experiments demonstrated that the proposed method can obtain accurate three-dimensional reconstructions for all 10 fingers.

obtained three-dimensional points computed without applying interpolation algorithms was 768 , and the distance between three-dimensional points along the $x$ and $y$ axes was $0.63 \mathrm{~mm}$.

\section{Performance of the proposed system}

This subsection presents tests performed to evaluate different aspects of the proposed system under different operating conditions. First, we evaluated the accuracy under standard operating conditions. Second, we analyzed the accuracy under different light conditions. Third, we examined the robustness to intentional misplacements of the finger. Finally, we analyzed the computational efficiency of the proposed algorithms.
1) Accuracy under standard operating conditions: We evaluated the performance of the proposed method under standard operating conditions using the samples from Dataset ${ }_{A}$.

The tests primarily focused on the rotation angle $\Delta_{R}$ and the number of three-dimensional rotations $n_{R}$ simulated in the enrollment phase because these variables can significantly affect the performance of the biometric system. The evaluated parameters were Receiver Operating Characteristic (ROC) curves [1], Equal Error Rate (EER) [104] and FMR 1000 (the lowest False Non-Match Rate for False Match Rate 0.1\%) [105].

We found that $n_{R}=9$ permitted satisfactory results from samples captured with correct finger placements, with an EER of $0.06 \%$. 
To study the effect of the number of three-dimensional rotations in the enrollment step, we tested different configurations of $n_{R}$. For convenience, we report the results in terms of computational time: $n_{R}=1$ (no rotations along the pitch and roll axes performed during the enrollment step); $n_{R}=9$ (iterative rotation of the three-dimensional finger models along the pitch and roll axes in all combinations of the angles $\left[-15^{\circ}, 0^{\circ},+15^{\circ}\right]$ in the enrollment step).

Fig. 17 presents the ROC curves obtained using Dataset ${ }_{A}$, and Table I provides the numerical error values. Because the matcher is non-symmetrical, every test regarded 16, 576 (296× $8 \times 7)$ genuine identity comparisons and 5, 588, $480(296 \times$ $295 \times 8 \times 8$ ) impostor identity comparisons.

Fig. 17 and Table I indicate that the accuracy obtained for Dataset $A_{A}$ by enrolling multi-templates corresponding to single templates $\left(n_{R}=1\right)$ is satisfactory for many biometric applications, with EER $=0.17 \%$.

Multi-templates composed of $n_{R}=9$ templates yielded the best performance, with EER $=0.06 \%$. These results demonstrate that multiple three-dimensional rotations can increase the accuracy of the biometric system and the robustness to uncontrolled finger orientations.

In the considered dataset, however, higher numbers of rotations $\left(n_{R}\right)$ did not result in significant accuracy improvements. For example, the proposed system achieved EER $=0.05 \%$ for $n_{R}=9$.

2) Robustness to environmental illumination: Samples in Dataset $_{A}$ were collected under different environmental light conditions (with artificial illumination and natural light at different hours of the day). The results presented in Fig. 17 and Table I show that the accuracy of the proposed method is not affected by changes in environmental light conditions. These variations, in fact, did not reduce the quality of the captured images and, consequently, the recognition accuracy of the proposed biometric system.

3) Sensitivity analysis: we performed a sensitivity analysis of the most relevant parameters of the proposed system on Dataset $_{A}$. Results showed that the system is robust to small variations of the parameters.

An important parameter consists in the angle $\Delta_{R}$ used to compute touch-compatible images. Small values require high numbers of rotations $n_{R}$ to overcome differences in the finger's placement, therefore increasing the computational time. High values can reduce the final accuracy of the system. We found that a good tradeoff is $\Delta_{R}=15^{\circ}$ and we performed the sensitivity analysis around this value. As an example, with $n_{R}=9$, a small variation of $5^{\circ}\left(\Delta_{R}=20^{\circ}\right)$ increased the EER from $0.06 \%$ to $0.07 \%$.

The second set of parameters that relevantly influence the system accuracy are the ones used to compute the threedimensional finger shape. We varied the values $s_{d}$ and $l$ of $\pm 20 \%$ of their optimal configuration and the value $w$ of $+20 \%$ and $+40 \%$. We did not reduce the value of $w$ since we empirically estimated that a value of \pm 70 pixels represents the minimum search range usable with the proposed acquisition setup. Within the tested range of parameters, the worst EER achieved with $n_{R}=1$ is $0.20 \%$ and the worst EER with $n_{R}=9$ is $0.07 \%$.

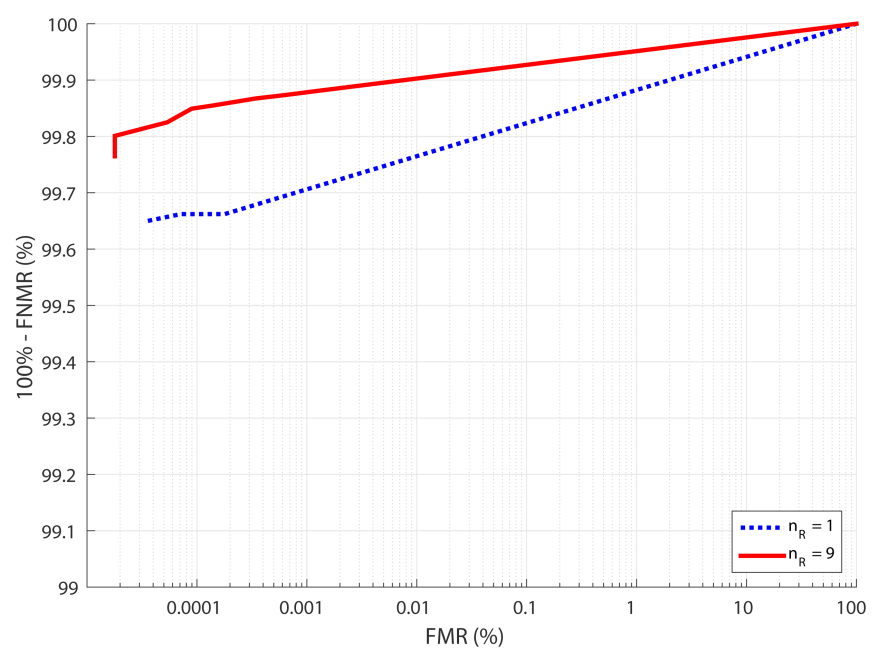

Fig. 17. ROC curves representing the accuracy of the proposed touchless system under standard operating conditions $\left(\right.$ Dataset $\left._{A}\right)$. The results represent different numbers of three-dimensional rotations $n_{R}$ performed during the enrollment step. Every test included 5, 605, 056 identity comparisons. The configuration that yielded the best accuracy was $n_{R}=9$, with EER $=0.06 \%$.

TABLE I

ACCURACY OF THE PROPOSED BIOMETRIC SYSTEM USING SAMPLES ACQUIRED UNDER STANDARD OPERATING CONDITIONS (Dataset ${ }_{A}$ ).

\begin{tabular}{ccc}
\hline \hline$n_{R}$ & FMR $_{1000}(\%)$ & EER (\%) \\
\hline 1 & 0.34 & 0.17 \\
9 & 0.12 & 0.06 \\
\hline \hline
\end{tabular}

4) Robustness to intentional misplacements and rotations: We evaluated the robustness of the proposed system in critical applications and uncollaborative contexts using samples appositely acquired with strong finger rotations $\left(\right.$ Dataset $\left._{B}\right)$. The system was able to properly compensate a great number of poor-quality acquisitions performed with high finger rotations. However, very high rotations in the roll and pitch directions reduced the capability of matching genuine samples due to the presence of out-of-focus regions, perspective deformations, and a reduced size of the ROI (central area of the fingertip). However, for intentional misplacements and rotations, the proposed system achieved EER $\leq 2 \%$ in its standard configuration.

In this test, we simulated an application scenario in which the enrollment phase is supervised and the verification phase is based on wrongly performed acquisitions. We therefore divided Dataset ${ }_{B}$ in two subsets.

- Dataset $_{B}$ enrollment: For each finger, 8 samples acquired with standard finger placements were used to compute the enrolled multi-templates.

- Dataset $_{B}$ probe: For each finger, 12 samples acquired with intentional misplacements and rotations of the finger were used to compute the probe templates.

For each of the considered configurations, we performed 5, 760 $(12 \times 8 \times 60)$ genuine identity comparisons and $339,840(12 \times$ $8 \times 60 \times 59)$ impostor identity comparisons. The results are presented in Table II. 
TABLE II

ACCURACY OF THE PROPOSED BIOMETRIC SYSTEM FOR SAMPLES ACQUIRED WITH INTENTIONAL MISPLACEMENTS AND ROTATIONS OF THE FINGER ( Dataset $\left._{B}\right)$.

\begin{tabular}{ccc}
\hline \hline$n_{R}$ & FMR $_{1000}(\boldsymbol{\%})$ & EER (\%) \\
\hline 1 & $7.95 \%$ & $3.98 \%$ \\
9 & $4.13 \%$ & $2.07 \%$ \\
25 & $2.87 \%$ & $1.43 \%$ \\
49 & $2.40 \%$ & $1.20 \%$ \\
\hline \hline
\end{tabular}

As shown in Table II, high numbers of three-dimensional rotations $n_{R}$ effectively reduced the effects of misplacements and rotations on system accuracy. The EER achieved with $n_{R}=1$ was $3.98 \%$, and the EER achieved with $n_{R}=49$ was $1.20 \%$. These results also suggest that the three-dimensional reconstruction approach produces accurate results for a wide range of finger rotations.

The results reported in Table II are not comparable to those reported in Fig. 17 and Table I because the results reported in Table II reflect data that were acquired with intentionally performed misplacements and rotations of the finger. By contrast, the results reported in Fig. 17 and Table I reflect acquisitions performed under standard conditions.

Rotations along different axes (Fig. 2) do not contribute in the same manner to decreased performance of the biometric system. Therefore, we analyzed the matching scores obtained using samples acquired with different rotations. Fig. 18 a presents boxplots of the matching scores obtained with the standard system configuration $\left(n_{R}=9\right)$. As a reference, Fig. $18 \mathrm{~b}$ presents the boxplots of the matching scores obtained under standard application conditions ( Dataset $_{A}$ ).

Fig. 18 shows that roll and pitch rotations decrease the matching scores in genuine comparisons. This decrease is due to the presence of out-of-focus regions, perspective deformations, and the reduced size of the ROI, which could only be mitigated by using more cameras to obtain three-dimensional models representing a wider area of the fingertip. The matching scores obtained by impostor identity comparisons were not significantly altered, and most were equal to 0 when the Neurotechnology VeriFinger matching algorithm was used. The acquisitions of Dataset ${ }_{B}$ were obtained by attempting to rotate the finger on a single axis and controlling the rotations in the other two axes to separately evaluate the effect of every possible rotation on performance.

5) Computational time: We evaluated the computational time required by every software module of the proposed system. With high probability, an optimized industrial implementation should permit the use of the proposed approach in real-time live applications.

We wrote the proposed algorithms for preprocessing, three-dimensional reconstruction and computation of touchcompatible images using Matlab (R2011b 64 bit), and the feature extraction and matching methods were written in $\mathrm{C} \#$ using the SDK provided by Neurotechnology. Tests were performed on an Intel Xeon $3.60 \mathrm{GHz}$ workstation with Windows 7 Professional 64 bit. Our implementations were not optimized in terms of computational complexity, and they did not use parallel computing strategies. (a)

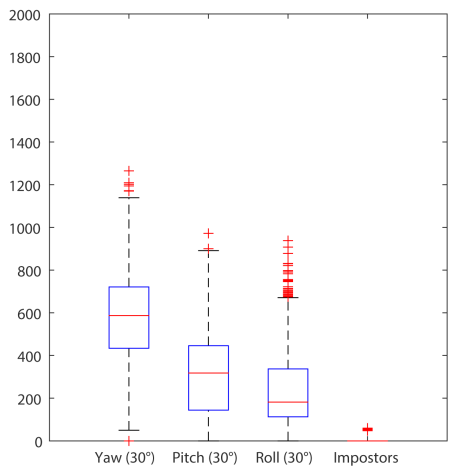

(b)

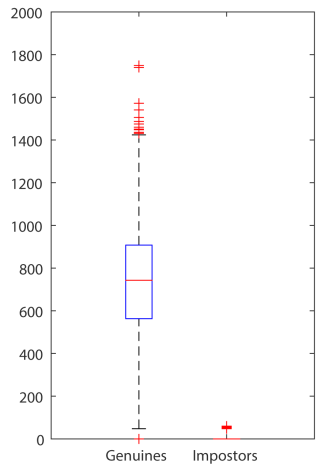

Fig. 18. Boxplot of the matching scores for samples acquired with intentional misplacements of the finger and samples captured under standard conditions: (a) matching scores reported for the considered finger rotation directions on samples acquired with intentional misplacements and rotations of the finger $\left(\right.$ Dataset $\left._{B}\right)$; (b) matching scores for samples captured under standard application conditions ( Dataset $_{A}$ ).

The proposed touchless technique first computes a touchcompatible fingerprint image and then performs the feature extraction and matching steps. The total time needed to compute a three-dimensional finger shape, a single touchcompatible image and a minutia template was 24.13 seconds. A large amount of this time (27.99\%) was devoted to matching pairs of corresponding points in the input images. For $n_{R}=1$, the matching algorithm required 0.11 seconds. For $n_{R}=9$, the matching algorithm required 1.07 seconds.

At enrollment, the system simulated rotations of the threedimensional shape of the finger, which is a computationally expensive task. Each rotation required 4.32 seconds. Because the enrollment is performed once, this task does not influence the computational efficiency of the system during its normal use.

All of the implemented algorithms are designed to be easily portable to parallel architectures. For example, a parallel implementation of these methods based on CUDA techniques [106] would drastically decrease the required computational time.

\section{Accuracy comparison with reference methods}

The accuracy of the proposed system was compared with that of touch-based systems and the most accurate touchless technologies reported in the literature. The results indicated better or similar performance compared with the considered biometric systems, with the advantage of using a fully touchless, on-the-move, less-constrained acquisition method. Moreover, the system achieved slightly better accuracy with respect to the reference touch-based technology for data acquired over a time period of one year; the proposed system achieved EER $=0.22 \%$, while the touch-based technology achieved $\mathrm{EER}=0.23 \%$.

1) Comparison with touch-based methods: To compare the performance of the proposed biometric recognition system with traditional touch-based technologies, we performed a scenario evaluation that considered a recognition application for access control in a laboratory. The accuracy of the proposed system was comparable to that of touch-based technologies. 
We compared the performance of our touchless system on Dataset $_{A}$ and the accuracy of the biometric recognition software VeriFinger on Dataset ${ }_{T}$. We used the operative configuration of the system $\left(n_{R}=9\right)$. The test performed on Dataset $_{A}$ included 16,576 $(296 \times 8 \times 7)$ genuine identity comparisons and 5,588, $480(296 \times 295 \times 8 \times 8)$ impostor identity comparisons, while the test performed on Dataset $_{T}$ included 3, $552(296 \times 4 \times 3)$ genuine identity comparisons and $1,397,120(296 \times 295 \times 4 \times 4)$ impostor identity comparisons related to samples captured from the same individuals included in Dataset $_{A}$.

The results presented in Table III demonstrate that the proposed system obtained a recognition accuracy comparable to that of the reference touch-based recognition method in the considered scenario. Moreover, the accuracy of the proposed system could be increased if the users became more proficient in using the proposed system. Experience in the use of sensors increases the recognition accuracy of biometric technologies.

We also evaluated the performance of the proposed touchless system and the reference touch-based technology using data acquired over one year. The proposed system exhibited stable performance during the considered time period, obtaining slightly better accuracy than the reference touch-based technology.

We tested the proposed system on Dataset $A Y$ (performing $148 \times 16 \times 15=35,520$ genuine identity comparisons and $148 \times 147 \times 16 \times 16=5,569,536$ impostor identity comparisons) and the touch-based system on Dataset ${ }_{A T}$ (performing $148 \times 8 \times 7=8,288$ genuine identity comparisons and $148 \times 147 \times 8 \times 8=1,392,384$ impostor identity comparisons). Table IV summarizes the results.

Table IV indicates that the performances of the compared biometric systems were similar. In particular, the proposed system achieved EER $=0.22 \%$ and the reference touch-based system achieved EER $=0.23 \%$.

2) Comparison with other touchless techniques: We compared the accuracy of the proposed system with that of other touchless recognition technologies in the literature. A direct performance comparison was not possible because all of the considered technologies are based on different acquisition hardware and processing methods that would be difficult to replicate in our laboratory. The results reported in the literature indicate that the accuracy of the proposed system is similar or superior to that of the most accurate touchless fingerprint recognition technologies, and the proposed system presents the advantage of reducing acquisition constraints.

A few studies have reported the accuracy of touchless fingerprint recognition technologies [39,50,55,59,75,83,85]. Most of these studies describe tests performed on datasets smaller than the datasets we used to evaluate our system. Moreover, most of the systems achieved an EER of approximately $1 \%$. Public tests of commercial devices [94] also report similar performance. [94] describes a test performed on a dataset of 1,800 samples acquired from 150 individuals using the touchless device TBS S120, with a resultant EER of approximately $0.5 \%$.
TABLE III

COMPARISON OF THE PROPOSED TOUCHLESS SYSTEM AND THE REFERENCE TOUCH-BASED TECHNOLOGIES, ON THE SAME USERS.

\begin{tabular}{lcc}
\hline \hline System & FMR $_{1000}(\%)$ & EER (\%) \\
\hline Proposed method & 0.12 & 0.06 \\
Touch-based system & 0.06 & 0.03 \\
\hline \hline
\end{tabular}

Notes: Implemented system $=$ results with configuration $n_{R}=9$ on Dataset $A$; Touch-based system $=$ results of the software VeriFinger on Dataset $T$.

TABLE IV

COMPARISON OF THE PROPOSED TOUCHLESS SYSTEM AND THE REFERENCE TOUCH - BASED TECHNOLOGIES ON SAMPLES ACQUIRED IN A TIME LAPSE OF ONE YEAR.

\begin{tabular}{lcc}
\hline \hline System & FMR $_{1000}(\%)$ & EER (\%) \\
\hline Proposed method & 0.43 & 0.22 \\
Touch-based system & 0.46 & 0.23 \\
\hline \hline
\end{tabular}

Note: Implemented system $=$ results with configuration $n_{R}=9$ on Dataset $_{T A}$; Touch-based system $=$ results of the software VeriFinger on Dataset $_{T Y}$

\section{E. User acceptability}

We performed a preliminary analysis of user acceptability and obtained satisfactory results.

After performing the biometric acquisitions using both the proposed touchless fingerprint recognition system and the reference touch-based technology, each volunteer was asked to complete an evaluation form. Table $\mathrm{V}$ summarizes relevant results for questions related to acceptability and user experience. Student's t test indicated that the differences in the evaluations were very significant.

Table V shows a positive response to the proposed system for most of the questions. In particular, $96.7 \%$ of the volunteers prefer the proposed touchless system to touch-based technologies, and $100 \%$ of the volunteers considered the proposed system more hygienic.

Moreover, we observed that users considered the proposed system more privacy-compliant. This perception may be due to the fact that no latent fingerprint can be left. In addition, the proposed system is significantly different from touch-based devices used by the police or border control authorities, thus reducing the feeling that the owner of the biometric system can associate biometric data with police records or proscription lists [107-109].

Therefore, we concluded that the proposed system is considered more acceptable by the analyzed set of users.

\section{F. Compatibility and interoperability with touch-based sys- tems}

We evaluated the compatibility of the proposed system with biometric software designed for touch-based fingerprint recognition and the interoperability of the computed touchcompatible images with touch-based samples. In this paper, the terms compatibility and interoperability have the following meanings:

- Compatibility is the capability of biometric data to be processed by systems other than the one that created the samples or templates. Specifically, we consider the term compatibility as the capability of the fingerprint image $E$ 
TABLE V

RESULTS OF THE QUESTIONNAIRE EVALUATING THE USER ACCEPTABILITY OF THE PROPOSED BIOMETRIC SYSTEM.

\begin{tabular}{|c|c|c|c|}
\hline \multirow[t]{2}{*}{ Question } & \multicolumn{3}{|c|}{ Answer } \\
\hline & Touchless & Touch & Equivalent \\
\hline $\begin{array}{l}\text { Which is the most comfortable acquisition } \\
\text { system? }\end{array}$ & $43.3 \%$ & $30.0 \%$ & $26.7 \%$ \\
\hline Which is the faster acquisition procedure? & $76.7 \%$ & $10.0 \%$ & $13.3 \%$ \\
\hline $\begin{array}{l}\text { Which system is better in terms of hygiene } \\
\text { issues? }\end{array}$ & $100.0 \%$ & $0.0 \%$ & $0.0 \%$ \\
\hline $\begin{array}{l}\text { Which is the most privacy compliant sys- } \\
\text { tem? }\end{array}$ & $60.0 \%$ & $3.3 \%$ & $36.7 \%$ \\
\hline $\begin{array}{l}\text { Do you prefer touchless systems to touch- } \\
\text { based systems? }\end{array}$ & $96.7 \%$ & $3.3 \%$ & $0.0 \%$ \\
\hline
\end{tabular}

computed by the proposed system to be fully processed by software from various vendors designed for touchbased images (e.g., algorithms for feature extraction and matching).

- Interoperability includes the concept of compatibility and is measured as the accuracy level obtained by matching images captured with different devices, in our case, images captured with the proposed touchless system and a state-of-the-art touch-based device. Specifically, we consider the term interoperability as the accuracy with which a fingerprint image $E$ is matched against touchbased samples using methods designed for touch-based images.

Our results demonstrated that the system is effectively compatible with feature extraction and matching algorithms designed for touch-based samples. In addition, the proposed system exhibited similar or better interoperability with touchbased samples compared with commercial touchless systems.

To evaluate the compatibility of the proposed system with software designed for touch-based samples, we compared the applicability of the software NIST NBIS [110] and the software Neurotechnology VeriFinger [95] to touchless samples of Dataset $_{A}$ and touch-based images of Dataset D $_{T}$. The proposed method was used in its simplest configuration, without simulating finger rotations during the enrollment phase $\left(n_{R}=1\right)$. Table VI reports the EER achieved for each test.

Table VI shows that the performance of the NIST NBIS software was lower than that of the Neurotechnology VeriFinger software. The accuracy decreasing was comparable for both the touchless and touch-based fingerprint datasets.

To evaluate the interoperability of touch-compatible images with touch-based databases, we performed a verification test in which the gallery database was composed only of multi-templates computed from touchless samples and probe templates were computed only from touch-based samples. We used all of the samples from Dataset $_{A}$ and Dataset $_{T}$. The test consisted of $2,803,712$ identity comparisons $(8 \times$ $296 \times 4 \times 296)$. The results indicated that the proposed method for simulating three-dimensional rotations increased the interoperability of the biometric system. The EER achieved with $n_{R}=1$ was $4.62 \%$, the EER achieved with $n_{R}=9$ was $2.23 \%$, and the EER achieved with $n_{R}=25$ was $2.00 \%$.

These results should be compared with those reported in public tests on commercial touchless systems [94], which
TABLE VI

COMPATIBILITY OF THE PROPOSED TOUCHLESS SYSTEM (WITHOUT SIMULATING THREE-DIMENSIONAL ROTATIONS) WITH THE SOFTWARE NIST NBIS. RESULTS ARE COMPARED WITH THAT OBTAINED BY THE REFERENCE TOUCH-BASED TECHNOLOGY.

\begin{tabular}{lcc}
\hline \hline Software & \multicolumn{2}{c}{ EER } \\
& Dataset $_{A}$ & Dataset $_{T}$ \\
\hline Neurotechnology VeriFinger & $0.17 \%$ & $0.03 \%$ \\
NIST NBIS & $1.48 \%$ & $1.14 \%$ \\
\hline \hline
\end{tabular}

Note: the method has been applied on Dataset $A$ in its simplest configuration, with a single temple stored during the enrollment phase $\left(n_{R}=1\right)$.

achieved EERs of approximately $5.67 \%$ by performing a similar test on a dataset of 1,800 samples acquired from 150 individuals using the touchless device TBS S120.

\section{COnCLusions}

We presented a novel touchless fingerprint recognition system based on three-dimensional models that permits lessconstrained, fully touchless, fast, on-the-move acquisitions. The system is based on a two-view setup, specific algorithms capable of obtaining accurate three-dimensional reconstructions of touchless fingerprints, methods for compensating three-dimensional rotations and translations in finger placement, and algorithms for computing touch-compatible images from three-dimensional models.

The performed experiments aimed to analyze the acceptability and usability of the biometric recognition system, its recognition performance, the effect of environmental conditions and finger misplacements, compatibility with existing biometric recognition software, and interoperability with touch-based samples.

The results of questionnaires completed by volunteers who participated in the collection of biometric datasets demonstrated that users perceive the proposed touchless system to be more hygienic and privacy compliant with respect to touchbased systems. Moreover, $96.7 \%$ of the users preferred the proposed system to touch-based technologies, indicating its superior acceptability compared with traditional fingerprint recognition systems. For these reasons, the biometric system can be considered an alternative to touch-based fingerprint biometrics for application contexts in which usability, privacy and hygiene are important concerns (e.g., hospitals, stadiums and public buildings).

The accuracy of the proposed fingerprint recognition system was evaluated using multiple datasets and was compared with that of state-of-the-art touch-based technologies in a scenario evaluation. Using a dataset of 2,368 samples acquired from 296 fingers in a single session, the proposed system achieved an EER of $0.06 \%$, and the compared touch-based technology achieved an EER of $0.03 \%$ for images acquired from the same users. Using a dataset composed of 2,368 samples acquired from 158 fingers over one year, the proposed system obtained slightly better accuracy than the reference touchbased technology, achieving an EER of $0.22 \%$ compared with an EER of $0.23 \%$.

The performed tests also indicate that the implemented system is robust to uncontrolled environmental illumination and can tolerate a wide range of finger orientations. 
Finally, we evaluated the compatibility of the proposed system with minutia-based algorithms designed for touchbased samples and its interoperability with sets of touchbased templates. Our results demonstrated that the proposed algorithms generate touch-compatible images that are compatible with different software applications. Evaluation of the interoperability with touch-based images demonstrated that the proposed system achieved accuracy superior or comparable to those of commercial touchless sensors.

Future work should include the study of optimized feature extraction and matching techniques and the ability to use both three-dimensional and two-dimensional characteristics.

\section{ACKNOWLEDGMENT}

This work was supported in part by: the EC within the 7FP under grant agreement 312797 (ABC4EU); the EC within the H2020 program under grant agreement 644597 (ESCUDOCLOUD); and the Italian Ministry of Research within the project "GenData 2020" (2010RTFWBH).

\section{REFERENCES}

[1] D. Maltoni, D. Maio, A. K. Jain, and S. Prabhakar, Handbook of Fingerprint Recognition, 2nd ed. Springer Publishing Company, Incorporated, 2009.

[2] R. Donida Labati and F. Scotti, "Fingerprint," in Encyclopedia of Cryptography and Security (2nd ed.), H. van Tilborg and S. Jajodia, Eds. Springer, 2011, pp. $460-465$.

[3] R. Donida Labati, V. Piuri, and F. Scotti, Touchless Fingerprint Biometrics, ser. Series in Security, Privacy and Trust. CRC Press, 2015.

[4] G. Parziale, "Touchless fingerprinting technology," in Advances in Biometrics, N. K. Ratha and V. Govindaraju, Eds. Springer London, 2008, pp. 25-48.

[5] R. Donida Labati, A. Genovese, V. Piuri, and F. Scotti, "Touchless fingerprint biometrics: a survey on $2 \mathrm{~d}$ and $3 \mathrm{~d}$ technologies," Journal of Internet Technology, vol. 15, no. 3, pp. 325 - 332, May 2014.

[6] A. de Santos-Sierra, C. Sánchez-Ávila, G. B. del Pozo, and J. GuerraCasanova, "Unconstrained and contactless hand geometry biometrics," Sensors, vol. 11, no. 11, pp. $10143-10164,2011$.

[7] V. Kanhangad, A. Kumar, and D. Zhang, "Contactless and pose invariant biometric identification using hand surface," IEEE Trans. on Image Processing, vol. 20, no. 5, pp. 1415-1424, May 2011.

[8] A. Genovese, V. Piuri, and F. Scotti, Touchless Palmprint Recognition Systems, ser. Advances in Information Security. Springer, 2014.

[9] Y. Han, Z. Sun, F. Wang, and T. Tan, "Palmprint recognition under unconstrained scenes," in Proc. of the 8th Asian Conference on Computer Vision - Volume Part II, 2007, pp. 1-11.

[10] W. Li, D. Zhang, D. Zhang, G. Lu, and J. Yan, "3-D palmprint recognition with joint line and orientation features," IEEE Trans. on Systems, Man, and Cybernetics, Part C: Applications and Reviews, vol. 41, no. 2, pp. 274-279, March 2011.

[11] W. Jia, R.-X. Hu, Y.-K. Lei, Y. Zhao, and J. Gui, "Histogram of oriented lines for palmprint recognition," IEEE Trans. on Systems, Man, and Cybernetics: Systems, vol. 44, no. 3, pp. 385-395, March 2014.

[12] S. Malassiotis, N. Aifanti, and M. Strintzis, "Personal authentication using 3-D finger geometry," IEEE Trans. on Information Forensics and Security, vol. 1, no. 1, pp. 12-21, March 2006.

[13] M.-K. Kim and P. Flynn, "Finger-knuckle-print verification based on vector consistency of corresponding interest points," in Proc. of the IEEE Winter Conf. on Applications of Computer Vision, March 2014, pp. 992-997.

[14] Q. Zhang, Y. Zhou, D. Wang, and X. Hu, "Personal authentication using hand vein and knuckle shape point cloud matching," in Proc. of the 2013 IEEE Sixth Int. Conf. on Biometrics: Theory, Applications and Systems (BTAS), September 2013, pp. 1-6.

[15] G. Medioni, J. Choi, C.-H. Kuo, and D. Fidaleo, "Identifying noncooperative subjects at a distance using face images and inferred three-dimensional face models," IEEE Trans. on Systems, Man and Cybernetics, Part A: Systems and Humans, vol. 39, no. 1, pp. 12-24, January 2009.
[16] X. Zhao, G. Evangelopoulos, D. Chu, S. Shah, and I. Kakadiaris, "Minimizing illumination differences for 3D to 2D face recognition using lighting maps," IEEE Trans. on Cybernetics, vol. 44, no. 5, pp. 725-736, May 2014.

[17] M. De Marsico, M. Nappi, D. Riccio, and H. Wechsler, "Robust face recognition for uncontrolled pose and illumination changes," IEEE Trans. on Systems, Man, and Cybernetics: Systems, vol. 43, no. 1, pp. 149-163, January 2013.

[18] R. Min, N. Kose, and J.-L. Dugelay, "KinectFaceDB: A kinect database for face recognition," IEEE Trans. on Systems, Man, and Cybernetics: Systems, vol. 44, no. 11, pp. 1534-1548, November 2014.

[19] M. De Marsico, M. Nappi, and D. Riccio, "Faro: Face recognition against occlusions and expression variations," IEEE Trans. on Systems, Man and Cybernetics, Part A: Systems and Humans, vol. 40, no. 1, pp. 121-132, January 2010.

[20] Y. Du, E. Arslanturk, Z. Zhou, and C. Belcher, "Video-based noncooperative iris image segmentation," IEEE Trans. on Systems, Man, and Cybernetics, Part B: Cybernetics, vol. 41, no. 1, pp. 64-74, February 2011.

[21] R. Donida Labati and F. Scotti, "Noisy iris segmentation with boundary regularization and reflections removal," Image and Vision Computing, Iris Images Segmentation Special Issue, vol. 28, no. 2, pp. 270 - 277, February 2010.

[22] R. Donida Labati, A. Genovese, V. Piuri, and F. Scotti, Iris segmentation: state of the art and innovative methods, ser. Intelligent Systems Reference Library, C. Liu and V. Mago, Eds. Springer, 2012, vol. 37.

[23] G. Rogez, J. Rihan, J. Guerrero, and C. Orrite, "Monocular 3-D gait tracking in surveillance scenes," IEEE Trans. on Cybernetics, vol. 44, no. 6, pp. 894-909, June 2014.

[24] J. Bustard and M. Nixon, "Toward unconstrained ear recognition from two-dimensional images," IEEE Trans. on Systems, Man and Cybernetics, Part A: Systems and Humans, vol. 40, no. 3, pp. 486494, May 2010.

[25] R. Donida Labati, A. Genovese, V. Piuri, and F. Scotti, "Contactless fingerprint recognition: a neural approach for perspective and rotation effects reduction," in Proc. IEEE Symposium on Computational Intelligence in Biometrics and Identity Management, April 2013, pp. 22-30.

[26] R. Donida Labati, V. Piuri, and F. Scotti, "Neural-based quality measurement of fingerprint images in contactless biometric systems," in Proc. of the 2010 Int. Joint Conf. on Neural Networks, July 2010, pp. $1-8$.

[27] D. Lee, K. Choi, H. Choi, and J. Kim, "Recognizable-image selection for fingerprint recognition with a mobile-device camera," IEEE Trans. on Systems, Man, and Cybernetics, Part B: Cybernetics, vol. 38, no. 1, pp. 233-243, February 2008.

[28] W. Kang and Q. Wu, "Pose-invariant hand shape recognition based on finger geometry," IEEE Trans. on Systems, Man, and Cybernetics: Systems, vol. 44, no. 11, pp. 1510-1521, November 2014.

[29] AFIS and I. Biometrics Consulting, "Fingerprint-on-the-move," http://www.afisandbiometrics.com.

[30] H. Proenca, E. Du, and J. Scharcanski, "Introduction to the special issue on unconstrained biometrics: advances and trends," Signal, Image and Video Processing, vol. 5, no. 4, pp. 399-400, 2011.

[31] G. Acampora and V. Loia, "A proposal of ubiquitous fuzzy computing for ambient intelligence," Information Sciences, vol. 178, no. 3, pp. 631-646, 2008.

[32] M. Hossain, A. Shirehjini, A. Alghamdi, and A. El Saddik, "Adaptive interaction support in ambient-aware environments based on quality of context information," Multimedia Tools and Applications, vol. 67, no. 2, pp. 409-432, 2013.

[33] G. Acampora, V. Loia, M. Nappi, and S. Ricciardi, "Ambient intelligence framework for context aware adaptive applications," in Proc. of the 7th International Workshop on Computer Architecture for Machine Perception, July 2005, pp. 327-332.

[34] P. K. Atrey, A. El-Saddik, and M. S. Kankanhalli, "Effective multimedia surveillance using a human-centric approach," Multimedia Tools Appl., vol. 51, no. 2, pp. 697-721, 2011.

[35] V. Ciriani, S. De Capitani di Vimercati, S. Foresti, and P. Samarati, "Theory of privacy and anonymity," in Algorithms and Theory of Computation Handbook (2nd edition), M. Atallah and M. Blanton, Eds. CRC Press, 2009.

[36] R. Donida Labati, A. Genovese, V. Piuri, and F. Scotti, "Fast 3-D fingertip reconstruction using a single two-view structured light acquisition," in Proc. 2011 IEEE Workshop on Biometric Measurements and Systems for Security and Medical Applications, September 2011, pp. $1-8$. 
[37] A. J. Mansfield and J. L. Wayman, Best Practices in Testing and Reporting Performance of Biometric Devices: Version 2.01, ser. NPL report. Centre for Mathematics and Scientific Computing, National Physical Laboratory, 2002.

[38] V. Piuri and F. Scotti, "Fingerprint biometrics via low-cost sensors and webcams," in Proc. of the 2nd IEEE Int. Conf. on Biometrics: Theory, Applications and Systems, October 2008, pp. 1-6.

[39] M. O. Derawi, B. Yang, and C. Busch, "Fingerprint recognition with embedded cameras on mobile phone," in MobiSec, R. Prasad, K. Farkas, A. U. Schmidt, A. Lioy, G. Russello, and F. L. Luccio, Eds., vol. 94. Springer, 2011, pp. 136-147.

[40] F. Han, J. Hu, M. Alkhathami, and K. Xi, "Compatibility of photographed images with touch-based fingerprint verification software," in Proc. of the IEEE Conf. on Industrial Electronics and Applications, June 2011, pp. 1034-1039.

[41] B. Hiew, A. Teoh, and D. Ngo, "Automatic digital camera based fingerprint image preprocessing," in Proc. of the Int. Conf. on Computer Graphics, Imaging and Visualisation, July 2006, pp. 182-189.

[42] B. Hiew, B. Andrew, and Y. Pang, "Digital camera based fingerprint recognition," in Proc. of the IEEE Int. Conf. on Telecommunications and Malaysia Int. Conf. on Communications, May 2007, pp. 676-681.

[43] B. Hiew, A. Teoh, and D. Ngo, "Preprocessing of fingerprint images captured with a digital camera," in Proc. of the Int. Conf. on Control, Automation, Robotics and Vision, December 2006, pp. 1-6.

[44] Y. Song, C. Lee, and J. Kim, "A new scheme for touchless fingerprint recognition system," in Proc. 2004 Int. Symposium on Intelligent Signal Processing and Communication Systems, November 2004, pp. 524527.

[45] L. Wang, R. H. A. El-Maksoud, J. M. Sasian, and V. S. Valencia, "Illumination scheme for high-contrast, contactless fingerprint images," in Novel Optical Systems Design and Optimization XII, R. J. Koshel and G. G. Gregory, Eds., vol. 7429, no. 1. SPIE, 2009.

[46] C. Lee, S. Lee, and J. Kim, "A study of touchless fingerprint recognition system," in Structural, Syntactic, and Statistical Pattern Recognition, ser. Lecture Notes in Computer Science, D.-Y. Yeung, J. Kwok, A. Fred, F. Roli, and D. de Ridder, Eds. Springer Berlin / Heidelberg, 2006, vol. 4109, pp. 358-365.

[47] L. Wang, R. H. A. El-Maksoud, J. M. Sasian, W. P. Kuhn, K. Gee, and V. S. Valencia, "A novel contactless aliveness-testing (CAT) fingerprint sensor," in Novel Optical Systems Design and Optimization XII, R. J. Koshel and G. G. Gregory, Eds., vol. 7429, no. 1. SPIE, 2009.

[48] E. Sano, T. Maeda, T. Nakamura, M. Shikai, K. Sakata, M. Matsushita, and K. Sasakawa, "Fingerprint authentication device based on optical characteristics inside a finger," in Proc. of the Conf. on Computer Vision and Pattern Recognition Workshop, June 2006, pp. 27-32.

[49] H. Choi, K. Choi, and J. Kim, "Mosaicing touchless and mirrorreflected fingerprint images," IEEE Trans. on Information Forensics and Security, vol. 5, no. 1, pp. 52-61, March 2010.

[50] F. Liu, D. Zhang, C. Song, and G. Lu, "Touchless multiview fingerprint acquisition and mosaicking," IEEE Trans. on Instrumentation and Measurement, vol. 62, no. 9, pp. 2492-2502, 2013.

[51] A. Pillai and S. Mil'shtein, "Can contactless fingerprints be compared to existing database?" in Proc. of the IEEE Conf. on Technologies for Homeland Security, 2012, pp. 390-394.

[52] C. Lee, S. Lee, J. Kim, and S.-J. Kim, "Preprocessing of a fingerprint image captured with a mobile camera," in Advances in Biometrics, ser. Lecture Notes in Computer Science, D. Zhang and A. Jain, Eds. Springer Berlin / Heidelberg, 2005, vol. 3832, pp. 348-355.

[53] L. Hong, Y. Wan, and A. Jain, "Fingerprint image enhancement: algorithm and performance evaluation," IEEE Trans. on Pattern Analysis and Machine Intelligence, vol. 20, no. 8, pp. 777-789, August 1998.

[54] M. Khalil and F. Wan, "A review of fingerprint pre-processing using a mobile phone," in Proc. of the 2012 Conf. on Wavelet Analysis and Pattern Recognition, 2012, pp. 152-157.

[55] B. Y. Hiew, A. B. J. Teoh, and O. S. Yin, "A secure digital camera based fingerprint verification system," J. of Visual Communication and Image Representation, vol. 21, no. 3, pp. 219-231, 2010.

[56] R. Donida Labati, V. Piuri, and F. Scotti, "A neural-based minutiae pair identification method for touchless fingeprint images," in Proc. IEEE Workshop on Computational Intelligence in Biometrics and Identity Management, April 2011, pp. 96-102.

[57] Q. Xiao, "Technology review - biometrics-technology, application, challenge, and computational intelligence solutions," IEEE Computational Intelligence Magazine, vol. 2, no. 2, pp. 5-25, May 2007.

[58] R. Donida Labati, A. Genovese, V. Piuri, and F. Scotti, "Measurement of the principal singular point in contact and contactless fingerprint images by using computational intelligence techniques," in Proc. of the IEEE Int. Conf. on Computational Intelligence for Measurement Systems and Applications, September 2010, pp. 18-23.

[59] D. Noh, H. Choi, and J. Kim, "Touchless sensor capturing five fingerprint images by one rotating camera," Optical Engineering, vol. 50, no. 11, pp. 113202-113 202-12, 2011.

[60] N. C. Francisco, A. Zaghetto, B. Macchiavello, E. A. B. da Silva, M. Lima-Marques, N. M. M. Rodrigues, and S. M. M. de Faria, "Compression of touchless multiview fingerprints," in Proc. IEEE Workshop on Biometric Measurements and Systems for Security and Medical Applications, September 2011, pp. 1-5.

[61] S. Mil'shtein, M. Baier, C. Granz, and P. Bustos, "Mobile system for fingerprinting and mapping of blood - vessels across a finger," in Proc. of the IEEE Conf. on Technologies for Homeland Security, May 2009, pp. 30-34.

[62] A. Kumar and Y. Zhou, "Human identification using finger images," IEEE Trans. on Image Processing, vol. 21, no. 4, pp. 2228-2244, April 2012.

[63] A. Kumar and Y. Zhou, "Contactless fingerprint identification using level zero features," in Proc. of the IEEE Computer Society Conf. on Computer Vision and Pattern Recognition Workshops, June 2011, pp. 114-119.

[64] IDAir, "ONEprint," http://www.idairco.com/wp-content/uploads/2012/ 11/ONEprint_1105.pdf.

[65] Mitsubishi, "Finger Identification Device By Penetrated Light," www.mitsubishielectric.com/company/rd/advance/pdf/vol115/vol115_ np1.pdf.

[66] TST Biometrics, "BiRD 3," http://www.bromba.com/tdbird3e.htm

[67] NEC, "Contactless Hybrid Finger Scanner - HS100-10," http://www.nec.com/en/global/solutions/biometrics/products/hybrid_ finger_scanner.html.

[68] IDAir, "AIRprint," http://www.idairco.com/wp-content/uploads/2012/ 11/AIRprint_1112.pdf.

[69] Morpho, "Finger-On-The-Fly," http://www.morpho.com/IMG/pdf/ EXT_FOTF_HD_EN_August_2014.pdf.

[70] G. Parziale and Y. Chen, "Advanced technologies for touchless fingerprint recognition," in Handbook of Remote Biometrics, ser. Advances in Pattern Recognition, M. Tistarelli, S. Z. Li, and R. Chellappa, Eds. Springer London, 2009, pp. 83-109.

[71] G. Parziale, E. Diaz-Santana, and R. Hauke, "The surround imager: A multi-camera touchless device to acquire 3D rolled-equivalent fingerprints." in Proc. of the Int. Conf. of Biometrics, 2006, pp. 244-250.

[72] G. Paar, M. d. Perucha, A. Bauer, and B. Nauschnegg, "Photogrammetric fingerprint unwrapping," J. of Applied Geodesy, vol. 2, pp. 13-20, 2008.

[73] F. Liu and D. Zhang, "3D fingerprint reconstruction system using feature correspondences and prior estimated finger model," Pattern Recognition, vol. 47, no. 1, pp. 178-193, 2014.

[74] Y. Wang, L. G. Hassebrook, and D. L. Lau, "Data acquisition and processing of 3-D fingerprints," IEEE Trans. on Information Forensics and Security, vol. 5, no. 4, pp. 750-760, December 2010.

[75] Y. Wang, L. G. Hassebrook, and D. L. Lau, "Noncontact, depth-detailed 3D fingerprinting," SPIE Newsroom, November 2009.

[76] D. Koller, L. Walchshäusl, G. Eggers, F. Neudel, U. Kursawe, P. Kühmstedt, M. Heinze, R. Ramm, C. Bräuer-Burchardt, G. Notni, R. Kafka, R. Neubert, H. Seibert, M. C. Neves, and A. Nouak, "3D capturing of fingerprints - on the way to a contactless certified sensor," in BIOSIG, 2011, pp. 33-44.

[77] S. Huang, Z. Zhang, Y. Zhao, J. Dai, C. Chen, Y. Xu, E. Zhang, and L. Xie, "3D fingerprint imaging system based on full-field fringe projection profilometry," Optics and Lasers in Engineering, vol. 52, pp. 123-130, 2014.

[78] W. Xie, Z. Song, and X. Zhang, "A novel photometric method for real-time 3D reconstruction of fingerprint," in Advances in Visual Computing, ser. Lecture Notes in Computer Science, G. Bebis, R. Boyle, B. Parvin, D. Koracin, R. Chung, R. Hammound, M. Hussain, T. KarHan, R. Crawfis, D. Thalmann, D. Kao, and L. Avila, Eds. Springer Berlin Heidelberg, 2010, vol. 6454, pp. 31-40.

[79] X. Pang, Z. Song, and W. Xie, "Extracting valley-ridge lines from point-cloud-based 3D fingerprint models," IEEE Computer Graphics and Applications, vol. 33, no. 4, pp. 73-81, July 2013.

[80] G. Abramovich, K. G. Harding, Q. Hu, S. Manickam, M. Ganesh, and C. A. Nafis, "Method and system for contactless fingerprint detection and verification," US20110064282 A1, March 2011.

[81] A. Baradarani, R. Maev, and F. Severin, "Resonance based analysis of acoustic waves for 3D deep-layer fingerprint reconstruction," in Proc. of the IEEE Int. Ultrasonics Symposium, July 2013, pp. 713-716. 
[82] M. J. Brooks, Shape from shading, B. K. P. Horn, Ed. Cambridge, MA, USA: MIT Press, 1989.

[83] A. Kumar and C. Kwong, "Towards contactless, low-cost and accurate 3D fingerprint identification," in Proc. of the IEEE Conf. on Computer Vision and Pattern Recognition, June 2013, pp. 3438-3443.

[84] Y. Chen, G. Parziale, E. Diaz-Santana, and A. K. Jain, "3D touchless fingerprints: Compatibility with legacy rolled images," in 2006 Biometrics Symposium, September 2006, pp. 1-6.

[85] Y. Wang, D. L. Lau, and L. G. Hassebrook, "Fit-sphere unwrapping and performance analysis of 3d fingerprints," Applied Optics, vol. 49, no. 4, pp. 592-600, February 2010.

[86] S. Shafaei, T. Inanc, and L. G. Hassebrook, "A new approach to unwrap a 3-D fingerprint to a 2-D rolled equivalent fingerprint," in Proc. of the IEEE 3rd Int. Conf. on Biometrics: Theory, Applications, and Systems, September 2009, pp. 1-5.

[87] Q. Zhao, A. Jain, and G. Abramovich, "3D to 2D fingerprints: Unrolling and distortion correction," in Proc. of the 2011 Int. Joint Conf. on Biometrics, October 2011, pp. 1-8.

[88] R. Donida Labati, A. Genovese, V. Piuri, and F. Scotti, "Virtual environment for 3-D synthetic fingerprints," in Proc. of the IEEE Int. Conf. on Virtual Environments, Human-Computer Interfaces and Measurement Systems, July 2012, pp. 48-53.

[89] R. Donida Labati, A. Genovese, V. Piuri, and F. Scotti, "Accurate 3D fingerprint virtual environment for biometric technology evaluations and experiment design," in Proc. of the IEEE Int. Conf. on Computational Intelligence and Virtual Environments for Measurement Systems and Applications, July 2013, pp. 43-48.

[90] R. Donida Labati, A. Genovese, V. Piuri, and F. Scotti, "Quality measurement of unwrapped three-dimensional fingerprints: a neural networks approach," in Proc. of the Int. Joint Conf. on Neural Networks, June 2012.

[91] S. Arora, K. Cao, A. Jain, and N. Paulter, "3d fingerprint phantoms," in Proc. of the 22nd Int. Conf. on Pattern Recognition, August 2014, pp. 684-689.

[92] Touchless Biometric Systems AG, "3D Terminal," http: //www.tbs-biometrics.com/fileadmin/tbs-media/products/3D-Terminal/ en_productsheet_3d-terminal.pdf.

[93] "Flashscan 3D," http://www.flashscan3d.com/technology/ fingerprinting.

[94] W. Zhou, J. Hu, I. Petersen, S. Wang, and M. Bennamoun, "A benchmark 3D fingerprint database," in 11th Int. Conf.on Fuzzy Systems and Knowledge Discovery, August 2014, pp. 935-940.

[95] Neurotechnology, "Verifinger SDK," http://www.neurotechnology.com/ verifinger.html.

[96] CROSSMATCH Technologies, $\quad$ Verifier http://www.neurotechnology.com.

[97] Z. Zhang, "A flexible new technique for camera calibration," IEEE Trans. on Pattern Analysis and Machine Intelligence, vol. 22, no. 11, pp. $1330-1334,2000$.

[98] J. Heikkila and O. Silvén, "A four-step camera calibration procedure with implicit image correction," Proc. of the IEEE Computer Society Conf. on Computer Vision and Pattern Recognition, pp. 1106-1112, 1997.

[99] M. Brown, D. Burschka, and G. Hager, "Advances in computational stereo," IEEE Trans. on Pattern Analysis and Machine Intelligence, vol. 25 , no. 8, pp. 993-1008, August 2003

[100] R. C. Gonzalez and R. E. Woods, Digital Image Processing (3rd Edition). Upper Saddle River, NJ, USA: Prentice-Hall, Inc., 2006.

[101] J. Jang and H. Kim, "Performance measures," in Encyclopedia of Biometrics, S. Li and A. Jain, Eds. Springer US, 2009, pp. 1062-1068.

[102] N. Boulgouris, K. Plataniotis, and E. Micheli-Tzanakou, Biometrics: Theory, Methods, and Applications, ser. IEEE Press Series on Computational Intelligence. Wiley, 2009.

[103] R. Guerchouche and F. Coldefy, "Camera calibration methods evaluation procedure for images rectification and 3D reconstruction," Orange Labs, France Telecom R \& D, 2008.

[104] D. Maio, D. Maltoni, R. Cappelli, J. Wayman, and A. Jain, "FVC2000: Fingerprint verification competition," IEEE Trans. on Pattern Analysis and Machine Intelligence, vol. 24, no. 3, pp. 402-412, March 2002.

[105] R. Cappelli, D. Maio, D. Maltoni, J. L. Wayman, and A. K. Jain, "Performance evaluation of fingerprint verification systems," IEEE Trans. on Pattern Analysis Mach. Intelligence, vol. 28, no. 1, pp. 3-18, January 2006.

[106] "NVIDIA CUDA Compute Unified Device Architecture - Programming Guide," http://docs.nvidia.com/cuda/cuda-c-programming-guide, 2007.
[107] R. Donida Labati, V. Piuri, and F. Scotti, Biometric privacy protection: guidelines and technologies, M. S. Obaidat, J. Sevillano, and F. Joaquim, Eds. Springer, 2012, vol. 314.

[108] S. Cimato, R. Sassi, and F. Scotti, "Biometrics and privacy," Recent Patents on Computer Science, vol. 1, pp. 98-109, June 2008.

[109] LLC International Biometric Group, "Bioprivacy initiative," 2003, http: //www.bioprivacy.org/.

[110] C. I. Watson, M. D. Garris, E. Tabassi, C. L. Wilson, R. M. Mccabe, S. Janet, and K. Ko, "User's guide to NIST biometric image software (NBIS)," 2007.

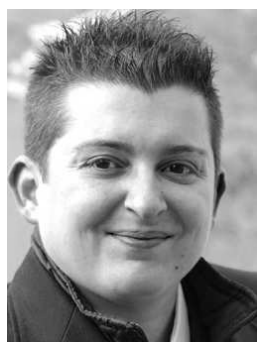

Ruggero Donida Labati is a Postdoctoral Research Assistant at the Università degli Studi di Milano, Italy. His research interests include: biometrics, signal and image processing, computational intelligence, and industrial applications. Original results have been published in $30+$ papers in international journals, proceedings of international conferences, books, and book chapters. http://www.di.unimi.it/donida

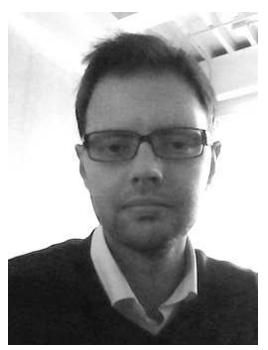

Angelo Genovese is a Postdoctoral Research Assistant at the Università degli Studi di Milano, Italy. His research interests include signal and image processing, three-dimensional reconstruction, computational intelligence technologies, biometric systems. He published $15+$ papers in international journals, proceedings of international conferences, books, and book chapters. http://www.di.unimi.it/genovese

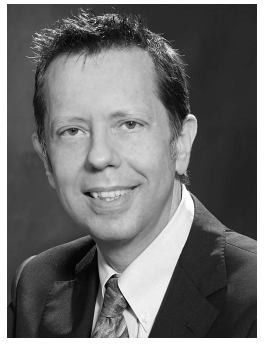

Vincenzo Piuri is a Full Professor in computer engineering at the Università degli Studi di Milano, Italy. His main research interests are: signal and image processing, biometrics, intelligent systems, digital and signal processing architectures. Original results have been published in $350+$ papers in international journals, proceedings of international conferences, books, and book chapters. He is Fellow of the IEEE, Distinguished Scientist of ACM, and Senior Member of INNS. He is IEEE Vice President for Technical Activities (2015). http://www.di.unimi.it/piuri

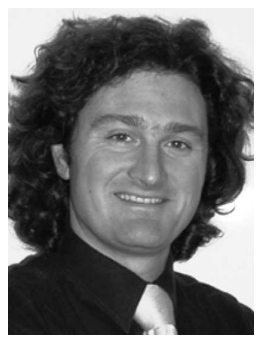

Fabio Scotti is an Associate Professor at the Università degli Studi di Milano, Italy. His research interests include: biometrics, signal and image processing, computational intelligence methods, industrial applications, high-level system design. He published 90+ papers in international journals, proceedings of international conferences, books, and book chapters. He is IEEE Senior Member. http://www.di.unimi.it/scotti 